\title{
Transport properties of a molecule embedded in an Aharonov-Bohm interferometer
}

\author{
Jong Soo Lim \\ Department de Física, Universitat de les Illes Balears, E-07122 Palma de Mallorca, Spain \\ and Department of Physics, Korea University, Anam-Dong Seongbuk-Gu, Seoul 136-701, Korea \\ Rosa López \\ Department de Física, Universitat de les Illes Balears, E-07122 Palma de Mallorca, Spain \\ and Instituto de Física Interdisciplinar y Sistemas Complejos (CSIC-UIB), E-07122 Palma de Mallorca, Spain
}

Gloria Platero

Teoría de la Materia Condensada, Instituto de Ciencia de Materiales de Madrid (CSIC), Cantoblanco, 28049 Madrid, Spain

Pascal Simon

Laboratoire de Physique des Solides, CNRS UMR-8502, Université Paris Sud, 91405 Orsay Cedex, France

(Received 28 October 2009; revised manuscript received 19 February 2010; published 13 April 2010)

\begin{abstract}
We theoretically investigate the transport properties of a molecule embedded in one arm of a mesoscopic Aharonov-Bohm interferometer. Due to the presence of phonons the molecule level position $\left(\varepsilon_{d}\right)$ and the electron-electron interaction $(U)$ undergo a polaronic shift, which affects dramatically the electronic transport through the molecular junction. When the electron-phonon interaction is weak the linear conductance presents Fano-line shapes as long as the direct channel between the electrodes is opened. The observed Fano resonances in the linear conductance are originated from the interference between the spin Kondo state and the direct path. For strong enough electron-phonon interaction, the electron-electron interaction is renormalized toward negative values, i.e., becomes effectively attractive. This scenario favors fluctuations between the empty and doubly occupied charge states and therefore promotes a charge Kondo effect. However, the direct path between the contacts breaks the electron-hole symmetry, which can efficiently suppress this charge Kondo effect. Nevertheless, we show that a proper tuning of the gate voltage is bale to revive the Kondo resonance. Our results are obtained by using the Numerical Renormalization approximation to compute the electronic spectral function and the linear conductance.
\end{abstract}

DOI: 10.1103/PhysRevB.81.165107

PACS number(s): 72.10.Fk, 73.63.-b, 73.23.-b

\section{INTRODUCTION}

Single-molecular transistors are seen as basic elements to build nanometer-scale electronic devices. ${ }^{1-3}$ Such nanostructures intermingle both the electronic and mechanical degrees of freedom and therefore give rise to physical scenarios. ${ }^{4-10}$ Among their advantages, they offer a tremendous diversity and potential functionalities. An archetype are the single- $C_{60}$ and $-C_{140}$ transistors in which single electron tunneling events are used to excite and probe the quantized mechanical degrees of freedom or vibrons of these molecules. ${ }^{4,8} \mathrm{Re}-$ cently, these features have been also observed in suspended carbon nanotubes. ${ }^{11,12}$ Importantly, these experiments show a rather strong electron-vibron coupling, which is consistent with the observation of the Franck-Condon blockade. ${ }^{12}$ The observation of a wide range of electron-vibron couplings in carbon nanotubes, i.e., from weak to strong couplings may motivate experiments to test many of the theoretical predictions in vibron-assisted transport through molecules. Furthermore, due to their flexibility, single molecules and especially carbon nanotubes can be contacted to nonmetallic leads such as ferromagnetic or superconducting leads and they can even exhibit many-body physics such as the Kondo effect when they are strongly coupled to the reservoirs. ${ }^{13,14}$

As we pointed out above, the electronic motion through single molecules is sensitive to vibrational modes that affect dramatically the current flow. ${ }^{4-10,15-24}$ The center-of-mass motion mode modulates asymmetrically the coupling between the molecule and may open new channels for transport. The observation of multiple features in the currentvoltage characteristic with identical spacing reflects the excitation of an integer number of a well-defined vibronic mode. ${ }^{4-11}$ Symmetric modes with an electron-vibron Holstein type of coupling may cause the Franck-Condon renormalization of the tunneling between the electrodes and the molecule. Such a mechanism suppresses the sequential and cotunneling transport channels through the molecule. ${ }^{15,16} \mathrm{Im}$ portantly, the electron-vibron interaction leads to the polaron shift in which the charging energy $U$ become effectively negative and the molecular level $\left(\varepsilon_{d}\right)$ is renormalized. ${ }^{19,25}$ As pointed out in Ref. 19, this intriguing aspect modifies the transport scenario opening a new transport channel through the molecular transistor. Owing to negative $U$, doubly occupied molecular states become energetically favored. Thus, when the gate voltage is properly adjusted so that the energy of the double occupancy is tuned to the energy of the empty state, the molecule is at "resonance" for the tunneling of electrons in pairs. ${ }^{19,22}$ This regime is known as the strong electron-vibron coupling regime. In chemistry, a variety of molecules in solution, have shown the feature known as potential inversion in analogy to the negative effective charging energy in the present context. ${ }^{26}$ In addition, the strong electron-vibron coupling regime can be achieved in vibrating 


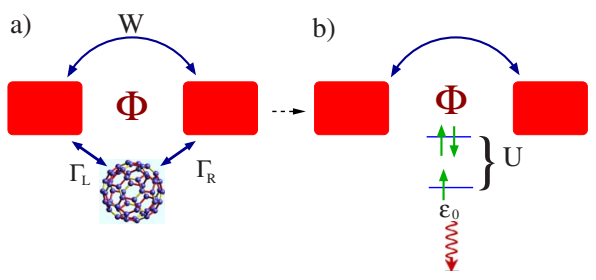

FIG. 1. (Color online). (a) Schematic of the molecule embedded in an Aharonov-Bohm interferometer. (b) Energy level diagram for the molecule.

carbon nanotube quantum dots as some experiments have already probed. ${ }^{11}$

The functionality of such devices depends greatly on the ability to preserve phase coherence through the molecule. ${ }^{27-30}$ Since the phase of electron wave function changes during the transport, it is of great interest to have access to the phase of the transmission amplitude in order to give a full characterization of the coherent transport through these devices. For this purpose, we investigate the widely known Aharonov-Bohm (AB) effect as a valuable tool to investigate quantum coherence of electrons. When the coherence of a circulating electron wave packet enclosing a magnetic flux $\Phi$ is preserved, this results in an extra fluxdependent phase shift. In the simplest realization of an $A B$ interferometer, an incoming electronic wave function splits into two paths, which join again into the outgoing electronic wave function. Applying a magnetic flux, which threads this closed geometry, the outgoing wave function acquires a fluxdependent phase, $\varphi=e \Phi / \hbar c$, where $\Phi=B S$ is the flux ( $B$ is the applied magnetic field, $S$ is the enclosing surface, and $e$ is the electron charge). As a consequence, the transmission is a periodic function of $\varphi$. The evolution of the electronic phase in $\mathrm{AB}$ geometries (ring and ringlike structures) was investigated in a series of pioneering works. ${ }^{31-34}$ Their theoretical predictions were tested in many type of experiments. ${ }^{35-44}$

In our particular case, we consider a molecular state occupied by an unpaired electron inserted in one of the arms of the $\mathrm{AB}$ interferometer. The other charge states with lower energies are doubly occupied and are therefore not active transport channels. The other arm acts as a direct path between the two electrodes (see Fig. 1). This situation corresponds to the case of single-molecules with a nonvanishing spin that behaves as magnetic impurities ${ }^{5}$ exhibiting manybody physics such as the Kondo effect. ${ }^{45}$ At sufficiently low temperatures, the localized spin residing in the molecule is able to form a many-body spin singlet state with the delocalized electrons in the electrodes. In this manner, the molecular spin is effectively screened and a narrow quasiparticle resonance emerges in the local density of states (DOS) at the molecule site. The width of such a singularity is related with the natural energy scale of this phenomenon given by the Kondo temperature, $T_{K}$. Thus, $T_{K}$ is a measure of the binding energy of the Kondo many-body singlet state. In transport through quantum dots, the Kondo effect leads to the unitary conductance as predicted theoretically ${ }^{46}$ and demonstrated experimentally. ${ }^{47}$ The unitary limit of the conductance in the Kondo regime is related to a transmission phase $\delta$ equal to $\pi / 2$. $^{45}$ This prediction has been tested by embedding a quan- tum dot in one of the arm of an Aharonov-Bohm ring. ${ }^{44,48}$ Theoretical studies addressing this problem have revealed the strong sensitivity of the phase shift to Kondo correlations. $49,50,52-57$ In our particular system we expect striking and interesting transport features due to the interplay of the electron-phonon (e-ph) coupling and Kondo correlations. In the Coulomb blockade regime, experiments performed with molecules such as $C_{60}$ and $C_{140}$ showed an internal mode of vibration of $\hbar \omega_{0} \approx 5-10 \mathrm{meV}$ (Refs. 4 and 8 ) while the vibron mode is on order $1 \mathrm{meV}$ in carbon nanotubes. ${ }^{12}$ This energy is comparable or even larger than the Kondo temperature observed in these molecules, which is on the order of few Kelvin. Additionally, for some molecules, the Coulomb charging energy becomes considerably reduced due to the effective screening coming from the electrodes. In this way, the electronic and some vibrational energy modes (typically on the order of $0.01-0.1 \mathrm{eV}$ ) may become comparable. This is, for example, the case where the interplay between the Kondo effect and the vibrons leads to a rich physical phenomena reflected in the molecular transport as shown below.

This paper is organized as follows: in Sec. II, we introduce the model Hamiltonian. We discuss different transport regimes depending on the strength of the e-ph coupling, the local mode frequency, $\omega_{0}$ and the electron-electron (e-e) onsite interaction $U$. In Sec. III, we briefly present our theoretical approach based on the numerical renormalization group (NRG). Expressions for the current and linear conductance are derived in Sec. IV. Our numerical results are detailed in Sec. V. Finally, we conclude this work by summarizing our main findings in Sec. VI. We add two Appendices that help in understanding the work. In Appendix A, we obtain the effective Hamiltonian for the different transport regimes. Appendix $\mathrm{B}$ is devoted to calculate explicitly the molecular Green's function and the phonon Green's function using the equation-of-motion approach.

\section{ANDERSON-LIKE AND KONDO-LIKE HAMILTONIANS}

In the following, we introduce the model Hamiltonian in order to describe the molecular transport through the Aharonov-Bohm interferometer. In a first step, we discuss the model we use to describe a isolated molecule. Next, we insert the molecule in the $\mathrm{AB}$ interferometer and analyze how the Kondo correlations emerge.

\section{A. Isolated molecule coupled to a single local phonon mode}

In order to describe the isolated molecule in the presence of vibrons, ${ }^{25}$ we use the following model Hamiltonian:

$$
\mathcal{H}_{\mathrm{Hol}}=\mathcal{H}_{M}+\mathcal{H}_{\mathrm{e}-\mathrm{ph}}+\mathcal{H}_{\mathrm{ph}},
$$

where

$$
\begin{gathered}
\mathcal{H}_{M}=\sum_{\sigma} \varepsilon_{d} d_{\sigma}^{\dagger} d_{\sigma}+U n_{d \uparrow} n_{d \downarrow}, \\
\mathcal{H}_{\mathrm{e}-\mathrm{ph}}=\lambda\left(a+a^{\dagger}\right)\left(n_{d}-1\right),
\end{gathered}
$$




$$
\mathcal{H}_{\mathrm{ph}}=\omega_{0} a^{\dagger} a .
$$

The isolated molecule is described by the Hamiltonian $\mathcal{H}_{M}$ : The operator $d_{\sigma}^{\dagger}\left(d_{\sigma}\right)$ is the creation (annihilation) operator of one electron in the unique active molecular orbital level $\left(\varepsilon_{d}\right)$ for transport. $U$ is the on-site Coulomb interaction, and $n_{d \sigma}$ $=d_{\sigma}^{\dagger} d_{\sigma}$ is the quantum occupation of the molecular level per spin $\left(n_{d}=\Sigma_{\sigma} n_{d \sigma}\right) \cdot a^{\dagger}(a)$ is the creation (annihilation) operator for an elementary quantized mechanical excitation or vibron, which we view here as a local phonon mode with a frequency $\omega_{0}$. In order to simplify our description of the e-ph interaction, we only account for single mode for phonons corresponding to any of the vibrational normal modes of the molecule. ${ }^{15-24}$ This is the extensively used Holstein model to describe in a simple fashion the observed molecular energy spectrum. ${ }^{25}$ This is a good approximation as long as the steplike features in the $I-V$ characteristic of some molecules are attributed to excitations of a well-defined single mode. ${ }^{4-11}$ The generalization to more modes is straightforward.

\section{First insight on the effect of the electron-phonon coupling}

In order to gain some first simple insight about the effect of the vibrational modes on the electronic properties, it is convenient to describe the phonon degrees of freedom by a simple harmonic oscillator potential coupled to the electrons. This can be written as

$$
\begin{aligned}
V(x)= & \frac{1}{2} m \omega_{0}^{2} x^{2}-\lambda\left(a^{\dagger}+a\right)\left(n_{d}-1\right)=\frac{1}{2} \omega_{0}\left(\frac{x}{\ell_{\mathrm{osc}}}\right)^{2}-\sqrt{2} \lambda\left(n_{d}\right. \\
& -1) \frac{x}{\ell_{\mathrm{osc}}}=\frac{1}{2} \omega_{0}\left(\frac{x}{\ell_{\mathrm{osc}}}-\frac{\sqrt{2}}{\omega_{0}} \lambda\left(n_{d}-1\right)\right)^{2}-\frac{\lambda^{2}}{\omega_{0}}\left(n_{d}-1\right)^{2},
\end{aligned}
$$

where

$$
\begin{gathered}
\ell_{\mathrm{osc}}=\frac{1}{\sqrt{m \omega_{0}}}, \\
x=\frac{1}{\sqrt{2 m \omega_{0}}}\left(a^{\dagger}+a\right) .
\end{gathered}
$$

Thus, the effects of the e-ph coupling leads to both a shift in the average position of the harmonic oscillator and an additional negative energy shift corresponding to the energy gain due to polaron-formation. By rewriting this polaronic shift, we can anticipate the resulting renormalizations

$$
-\frac{\lambda^{2}}{\omega_{0}}\left(n_{d}-1\right)^{2}=-\frac{\lambda^{2}}{\omega_{0}} n_{d}\left(n_{d}-1\right)+\frac{\lambda^{2}}{\omega_{0}} n_{d} .
$$

Reabsorbing these terms into the original Hamiltonian, we obtain the renormalizations

$$
U_{\mathrm{eff}}=U-\frac{2 \lambda^{2}}{\omega_{0}}, \quad \varepsilon_{d, \mathrm{eff}}=\varepsilon_{d}+\frac{\lambda^{2}}{\omega_{0}} .
$$

Note that the potential minimum position now depends on the occupation number of the dot. The renormalization of the e-e interaction gives rise to very different physical scenarios depending on the e-ph coupling $\lambda$. From Eq. (2.6), we see that $U_{\text {eff }}$ can be negative when $2 \lambda^{2} /\left(U \omega_{0}\right)>1$. This corresponds to the so-called strong e-ph coupling regime where the e-ph interaction induces an effective attraction between electrons. On the other hand, for a weak e-ph coupling, i.e., when $2 \lambda^{2} /\left(U \omega_{0}\right)<1$, the bipolaron quasiparticle is not energetically favorable since the induced effective e-e interaction remains positive.

\section{B. Including the leads: Kondo correlations}

Let us assume that a molecule is attached to the source and drain electrodes and placed in the lower arm of an Aharonov-Bohm interferometer (see Fig. 1). The upper arm connects directly the source and drain contacts by a direct path. The simplest Hamiltonian able to describe the system is the Anderson-Holstein Hamiltonian

$$
\mathcal{H}=\mathcal{H}_{C}+\mathcal{H}_{\mathrm{Hol}}+\mathcal{H}_{T}
$$

where

$$
\begin{gathered}
\mathcal{H}_{C}=\sum_{\alpha=L / R, k, \sigma} \varepsilon_{k} c_{\alpha k \sigma}^{\dagger} c_{\alpha k \sigma}, \\
+\sum_{k, k^{\prime}, \sigma}\left(W e^{i \varphi} c_{R k^{\prime} \sigma}^{\dagger} c_{L k \sigma}+\text { h.c. }\right), \\
\mathcal{H}_{T}=\sum_{\alpha=L / R, k, \sigma}\left(V_{\alpha} c_{\alpha k \sigma}^{\dagger} d_{\sigma}+\text { h.c. }\right),
\end{gathered}
$$

where we have defined $c_{L(R), k, \sigma}^{\dagger}\left[c_{L(R), k, \sigma}\right]$ is the creation (annihilation) operator for an electron in the state $k$ with spin $\sigma$ in the lead $L(R)$. We have assumed in our analysis that the leads are nonpolarized such that the dispersion relation $\varepsilon_{k}$ is spin independent. $V_{\alpha}$ is the tunneling amplitude for the $\alpha$ electrode with the molecule. $W$ is the amplitude probability for electron transfer from the left electrode to the right one, and $\varphi=2 \pi \Phi / \phi_{0}$ is the Aharonov-Bohm phase, with $\phi_{0}$ $=h c / e$ being the quantum flux

Kondo physics arises when the molecule is strongly contacted to the reservoirs. ${ }^{5,45}$ High-order tunneling processes between the delocalized electrons in the contacts and the localized electron in the molecule lead to screen effectively the molecular spin and to create the Kondo state. Then, a spin singlet many-body state is formed by the conduction band electrons and the localized unpaired electron in the molecule. As a result, the molecular DOS shows a very narrow peak at the Fermi energy $\left(E_{F}\right) .{ }^{45}$ This is the so-called Kondo resonance. In order to see how Kondo physics emerges in the presence of e-ph interaction, let us first perform a series of transformations of $\mathcal{H}$.

The first step consists in eliminating the e-ph coupling in $\mathcal{H}_{M}$ by applying the Lang-Firsov canonical transformation $^{58,59}$ to the full Hamiltonian $\tilde{\mathcal{H}} \rightarrow \mathcal{U}^{\dagger} \mathcal{H} \mathcal{U}$ where the operator $\mathcal{U}^{\dagger}$ is defined as

$$
\mathcal{U}^{\dagger}=\exp \left[\frac{\lambda}{\omega_{0}}\left(n_{d}-1\right)\left(a^{\dagger}-a\right)\right] \text {. }
$$

The resulting Hamiltonian $\tilde{\mathcal{H}}=\tilde{\mathcal{H}}_{C}+\tilde{\mathcal{H}}_{M}+\tilde{\mathcal{H}}_{T}$ reads in the 
parity basis

$$
\begin{gathered}
\tilde{\mathcal{H}}_{C}=\sum_{\ell=e / o, k, \sigma} \varepsilon_{k \sigma} c_{\ell k \sigma}^{\dagger} c_{\ell k \sigma}+\sum_{k, k^{\prime}, \sigma} W\left(c_{e k^{\prime} \sigma}^{\dagger} c_{e k \sigma}-c_{o k^{\prime} \sigma}^{\dagger} c_{o k \sigma}\right), \\
\tilde{\mathcal{H}}_{M}=\omega_{0} a^{\dagger} a+\left(\varepsilon_{d}+\frac{\lambda^{2}}{\omega_{0}}\right) n_{d}+\left(U-\frac{2 \lambda^{2}}{\omega_{0}}\right) n_{d \uparrow^{\top}} n_{d \downarrow}, \\
\tilde{\mathcal{H}}_{T}=\sqrt{2} V \sum_{\ell, k, \sigma}\left[e^{-\lambda\left(a^{\dagger}-a\right) / \omega_{0}} \cos (\varphi / 2) c_{e k \sigma}^{\dagger} d_{\sigma}\right. \\
\left.+e^{-\lambda\left(a^{\dagger}-a\right) / \omega_{0}} \sin (\varphi / 2) c_{o k \sigma}^{\dagger} d_{\sigma}+\text { h.c. }\right]
\end{gathered}
$$

where we have defined the even-odd parity basis as

$$
\begin{gathered}
c_{e k \sigma}=\frac{1}{\sqrt{2}}\left(e^{i \varphi / 2} c_{L k \sigma}+e^{-i \varphi / 2} c_{R k \sigma}\right), \\
c_{o k \sigma}=\frac{i}{\sqrt{2}}\left(-e^{i \varphi / 2} c_{L k \sigma}+e^{-i \varphi / 2} c_{R k \sigma}\right),
\end{gathered}
$$

and $V_{L}=V_{R} \equiv V$. Notice that now the vibronic degrees of freedom appear in a nontrivial manner in the tunneling part of the Hamiltonian. As we anticipated in Sec. I, the charging energy and the molecular level position are renormalized by the phonon frequency and the e-ph coupling as $U_{\text {eff }}=U$ $-2 \lambda^{2} / \omega_{0}$ and $\varepsilon_{d \text {,eff }}=\varepsilon_{d}+\lambda^{2} / \omega_{0}$. Due to this renormalization $U_{\text {eff }}$ can be negative inducing a bipolaronic attraction between the electrons [when $2 \lambda^{2} /\left(\omega_{0} U\right)>1$ ]. In this case and when $\Delta=\varepsilon_{d}+U=0$ (that corresponds to the particle hole or symmetric case), the zero and the doubly occupied molecule states are degenerate and the low-energy excitations consists of charge fluctuations with a large gap for the spin fluctuations. These energy excitations can be described in terms of an effective Kondo model in the charge sector where the molecular charge fluctuates between $n_{d}=0$ and $n_{d}=2$. On the contrary, in the weak e-ph coupling limit [when $2 \lambda^{2} /\left(\omega_{0} U\right)$ $<1]$ the magnitude of the e-e interaction is effectively reduced but it does not favor the bipolaron formation. Here, the physics corresponds to the common spin-1/2 Kondo effect where the singly occupied molecular state is the ground state. Let us detail both regimes.

\section{Weak electron-phonon coupling limit: $2 \lambda^{2} /\left(\omega_{0} U\right)<1$}

To treat this limit, we first perform a second order Raileigh-Schrödinger perturbative calculation (or projection method) in the tunneling Hamiltonian $\widetilde{\mathcal{H}}_{T}$, we derive an effective Kondo-type Hamiltonian of the form ${ }^{45}$

$$
\mathcal{H}_{\text {eff }}=\mathcal{H}_{10} \frac{1}{E_{1,0}-E_{0, m}} \mathcal{H}_{01}+\mathcal{H}_{12} \frac{1}{E_{1,0}-E_{2, m}} \mathcal{H}_{21} .
$$

The eigenenergies of the isolated molecule are given in Appendix A. The projectors read

$$
\mathcal{H}_{01}=\sqrt{2} \sum_{\ell, k, \sigma, m} V_{\ell}\langle m|\mathcal{U}| 0\rangle c_{\ell k \sigma}^{\dagger} d_{\sigma}\left(1-n_{d \bar{\sigma}}\right)
$$

$$
\mathcal{H}_{21}=\sqrt{2} \sum_{\ell, k, \sigma, m} V_{\ell}^{*}\left\langle m\left|\mathcal{U}^{+}\right| 0\right\rangle \operatorname{sgn}(\sigma) d_{\sigma}^{\dagger} n_{d \bar{\sigma}} c_{\ell k \sigma},
$$

where we have introduced

$$
\mathcal{U}^{ \pm}=\exp \left[ \pm \lambda\left(a^{\dagger}-a\right) / \omega_{0}\right]
$$

and defined $\bar{\sigma}=-\sigma, V_{e}=\cos (\varphi / 2) V$, and $V_{o}=\sin (\varphi / 2) V$. The explicit calculation of $\mathcal{H}_{\text {eff }}$ is given in Appendix A. After some algebra, the effective Kondo Hamiltonian can be rewritten in a more standard form as [see Eq. (A11)]

$$
\begin{aligned}
\mathcal{H}_{\mathrm{eff}}= & \sum_{\alpha, \beta, p, q} J^{\alpha \beta}\left[S^{z} \cdot\left(c_{\alpha p \uparrow}^{\dagger} c_{\beta q \uparrow}-c_{\alpha p \downarrow}^{\dagger} c_{\beta q \downarrow}\right)+S^{+} \cdot c_{\alpha p \downarrow}^{\dagger} c_{\beta q \uparrow}\right. \\
& \left.+S^{-} \cdot c_{\alpha p \uparrow}^{\dagger} c_{\beta q \downarrow}\right]+\frac{1}{2} \sum_{\alpha, \beta, p, q, \sigma} K^{\alpha \beta} c_{\alpha p \sigma}^{\dagger} c_{\beta q \sigma},
\end{aligned}
$$

where the first two lines are the Kondo interaction part while the last line corresponds to the potential scattering part characterized by the coupling $K^{\alpha \beta}$. Notice that the Kondo part is described by the isotropic Kondo model that is characterized by a single exchange coupling [see Eqs. (A7) and (A8) in Appendix A]

$$
\begin{aligned}
J^{\alpha \beta}= & J_{\|}^{\alpha \beta}=J_{\perp}^{\alpha \beta}=-2 \sum_{m}\left(\frac{V_{\alpha}^{*} V_{\beta}\left|\left\langle m\left|\mathcal{U}^{-}\right| 0\right\rangle\right|^{2}}{\frac{\lambda^{2}}{\omega_{0}}+\varepsilon_{d}-m \omega_{0}}\right. \\
& \left.+\sum_{m} \frac{V_{\alpha} V_{\beta}^{*}\left|\left\langle m\left|\mathcal{U}^{+}\right| 0\right\rangle\right|^{2}}{\frac{\lambda^{2}}{\omega_{0}}-\varepsilon_{d}-U-m \omega_{0}}\right),
\end{aligned}
$$

with

$$
\begin{gathered}
\left\langle m\left|\mathcal{U}^{ \pm}\right| 0\right\rangle=\exp \left[-\frac{1}{2}\left(\frac{\lambda}{\omega_{0}}\right)^{2}\right] \frac{1}{\sqrt{m !}}\left( \pm \frac{\lambda}{\omega_{0}}\right)^{m}, \\
|m\rangle=\frac{1}{\sqrt{m !}}\left(a^{\dagger}\right)^{m}|0\rangle .
\end{gathered}
$$

To shorten the notation, we omit the subindex ph in the molecular states. From Eq. (2.14), one has $\mathcal{U}^{-}=(-1)^{m} \mathcal{U}^{+}$and for the symmetric model, $\varepsilon_{d}=-U / 2$, Eq. (2.16) becomes

$$
J^{\alpha \beta}(\lambda)=\frac{8}{U} \sum_{m} \frac{V_{\alpha} V_{\beta}\left|\left\langle m\left|\mathcal{U}^{-}\right| 0\right\rangle\right|^{2}}{1-\frac{2 \lambda^{2}}{\omega_{0} U}+\frac{2 m \omega_{0}}{U}},
$$

where

$$
J^{o o}=J \cos ^{2}(\varphi / 2), \quad J^{o o}=J \sin ^{2}(\varphi / 2), \quad J^{e o}=\frac{J}{2} \sin (\varphi),
$$

with 


$$
J=\frac{8 V^{2}}{U} \sum_{m} \frac{|\langle m \mid \mathcal{U}-0\rangle|^{2}}{1-\frac{2 \lambda^{2}}{\omega_{0} U}+\frac{2 m \omega_{0}}{U}} .
$$

Expanding $J^{\alpha \beta}(\lambda)$ around $\lambda=0$, we find

$$
J^{\alpha \beta}(\lambda) \approx \frac{8}{U} V_{\alpha} V_{\beta}\left[1+2\left(1+\frac{U}{2 \omega_{0}}\right)^{-1} \frac{\lambda^{2}}{U \omega_{0}}\right] .
$$

Then, using the definition for the Kondo temperature in the isotropic Kondo model, one has

$$
T_{K} \propto \exp \left[-\frac{1}{J(\lambda) \rho_{0}}\right] .
$$

As a result, the e-ph coupling leads to an increase in the Kondo temperature in the weak e-ph regime as already found in Ref. 17 by Cornaglia and co-workers.

\section{Strong electron-phonon coupling: $2 \lambda^{2} /\left(\omega_{0} U\right)>1$}

In this regime, the e-ph interaction induces a bipolaronic attraction between electrons since $U_{\text {eff }}$ becomes negative. In this case, the ground state is a molecular doublet state composed of the doubly occupied molecular state $|2,0\rangle$ and the empty molecular state $|0,0\rangle$, which are degenerate. The lowenergy excitations thus consist of charge fluctuations and can be described by an effective Kondo model in which the role of spin is played now by a pseudospin variable that represents the two states of the doublet. Importantly, there is no rotational invariance in pseudospin space and therefore the effective model corresponds to an anisotropic Kondo model with different couplings, namely, $J_{\|}$and $J_{\perp}$. In order to gain some insights about the physical properties of this regime, we employ again the projection method. Before this, it is convenient to map the negative- $U$ Anderson-Holtstein model to an equivalent model with positive interaction. This can be carried out by using a particular particle-hole (p-h) transformation. ${ }^{60,61}$ First, we introduce new operators $\widetilde{d}, \widetilde{c}$ via

$$
\begin{gathered}
d_{\downarrow} \equiv-\widetilde{d}_{\downarrow}^{\dagger}, \quad c_{L k \downarrow} \equiv \tilde{c}_{2 \tilde{k} \downarrow}^{\dagger}, \quad c_{R k \downarrow} \equiv \tilde{c}_{1 \tilde{k} \downarrow}^{\dagger}, \\
d_{\uparrow} \equiv \tilde{d}_{\uparrow}, \quad c_{L k \uparrow} \equiv \widetilde{c}_{1 k \uparrow}, \quad c_{R k \uparrow} \equiv \widetilde{c}_{2 k \uparrow} .
\end{gathered}
$$

The p-h transformation yields $\varepsilon_{d \sigma} \rightarrow \widetilde{\varepsilon}_{d \sigma}=U / 2+\operatorname{sgn}(\sigma) \Delta / 2$ and $U \rightarrow \tilde{U}=-U$ where $\Delta=2 \varepsilon_{d}+U$ and $\varepsilon_{k \sigma}=-\varepsilon_{k \sigma}^{\tilde{k}}$ assuming the symmetry condition for the conduction band. The tunneling amplitudes are given by

$$
\begin{array}{ll}
\tilde{V}_{1 k \uparrow}=V_{L k \uparrow}, & \tilde{V}_{1 k \downarrow}=V_{R \tilde{k} \downarrow}^{*}, \\
\tilde{V}_{2 k \uparrow}=V_{R k \uparrow}, & \tilde{V}_{2 k \downarrow}=V_{L \tilde{k} \downarrow}^{*} .
\end{array}
$$

The $\mathrm{p}$-h transformation maps the electronic states of the molecule to the ones of the original model in this manner

$$
|0\rangle \mapsto|\widetilde{\downarrow}\rangle, \quad|2\rangle \mapsto|\widetilde{\uparrow}\rangle, \quad|\downarrow\rangle \mapsto|\widetilde{0}\rangle, \quad|\uparrow\rangle \mapsto|\widetilde{2}\rangle .
$$

Now, we perform the polaronic transformation ${ }^{58,59}$ combined with a canonical transformation such that the full Hamil- tonian in the parity basis [see Eq. (2.11)] reads (see Appen$\operatorname{dix}$ A)

$$
\begin{aligned}
& \tilde{\mathcal{H}}_{C}=\sum_{\ell=e / o, k, \sigma} \varepsilon_{k \sigma} \widetilde{c}_{\ell k \sigma}^{\dagger}, \widetilde{c}_{\ell k \sigma}+\sum_{k, k^{\prime}, \sigma} \operatorname{sgn}(\sigma) W\left(\widetilde{c}_{e k^{\prime} \sigma}^{\dagger} \widetilde{c}_{e k \sigma}\right. \\
& \left.-\widetilde{c}_{o k^{\prime} \sigma}^{\dagger} \widetilde{c}_{o k \sigma}\right) \text {, } \\
& \tilde{\mathcal{H}}_{M}=\sum_{\sigma}\left(\widetilde{\varepsilon}_{d \sigma}-\frac{\lambda^{2}}{\omega_{0}}\right) \tilde{d}_{\sigma}^{\dagger} \tilde{d}_{\sigma}+\left(\tilde{U}+\frac{2 \lambda^{2}}{\omega_{0}}\right) \tilde{n}_{d \uparrow} \tilde{n}_{d \downarrow}+\omega_{0} a^{\dagger} a, \\
& \widetilde{\mathcal{H}}_{T}=\sqrt{2} \sum_{\ell=e / o, k, \sigma}\left(\widetilde{V}_{\sigma} e^{-\operatorname{sgn}(\sigma) \lambda / \omega_{0}\left(a^{\dagger}-a\right)} \cos (\varphi / 2) \widetilde{c}_{e k \sigma}^{\dagger} \tilde{d}_{\sigma}\right. \\
& \left.+\widetilde{V}_{\sigma} e^{-\operatorname{sgn}(\sigma) \lambda / \omega_{0}\left(a^{\dagger}-a\right)} \sin (\varphi / 2) \widetilde{c}_{o k \sigma}^{\dagger} \tilde{d}_{\sigma}+\text { h.c. }\right),
\end{aligned}
$$

where we assumed $\widetilde{V}_{1 / 2 \sigma}=\widetilde{V}_{\sigma}$. The final step consists in obtaining an effective Hamiltonian by using again the projection method. We employ Eq. (2.12) and we refer to Appendix A for details

$$
\begin{aligned}
& \mathcal{H}_{01}=\sqrt{2} \sum_{\ell, k, \sigma, m} \tilde{V}_{\ell k \sigma}\left\langle m\left|\mathcal{U}^{-\operatorname{sgn}(\sigma)}\right| 0\right\rangle \widetilde{c}_{\ell k \sigma}^{\dagger} \tilde{d}_{\sigma}\left(1-\widetilde{n}_{d \bar{\sigma}}\right), \\
& \mathcal{H}_{21}=\sqrt{2} \sum_{\ell, k, \sigma, m} \tilde{V}_{\ell k \sigma}^{*}\left\langle m\left|\mathcal{U}^{+\operatorname{sgn}(\sigma)}\right| 0\right\rangle \operatorname{sgn}(\sigma) \tilde{d}_{\sigma}^{\dagger} \tilde{n}_{d \bar{c}} \widetilde{c}_{\ell k \sigma},
\end{aligned}
$$

with $\bar{\sigma}=-\sigma$, and $\tilde{V}_{e \sigma}=\cos (\varphi / 2) \tilde{V}_{\sigma}$ and $\tilde{V}_{o \sigma}=\sin (\varphi / 2) \tilde{V}_{\sigma}$. After the projection, the effective Hamiltonian $\mathcal{H}_{\text {eff }}$ corresponds to the anisotropic Kondo model with the following exchange couplings (see Appendix A)

$$
\begin{gathered}
J_{\|}^{\alpha \beta}=\frac{8}{U} \sum_{m} \frac{V_{\alpha} V_{\beta}\left|\left\langle m\left|\mathcal{U}^{-}\right| 0\right\rangle\right|^{2}}{\frac{2 \lambda^{2}}{\omega_{0} U}-1+\frac{2 m \omega_{0}}{U}}, \\
J_{\perp}^{\alpha \beta}=\frac{8}{U} \sum_{m} \frac{V_{\alpha} V_{\beta}\left\langle 0\left|\mathcal{U}^{-}\right| m\right\rangle\left\langle m\left|\mathcal{U}^{-}\right| 0\right\rangle}{\frac{2 \lambda^{2}}{\omega_{0} U}-1+\frac{2 m \omega_{0}}{U}}, \\
=\frac{8}{U} \sum_{m}(-1)^{m} \frac{V_{\alpha} V_{\beta}\left|\left\langle m\left|\mathcal{U}^{-}\right| 0\right\rangle\right|^{2}}{\frac{2 \lambda^{2}}{\omega_{0} U}-1+\frac{2 m \omega_{0}}{U}} .
\end{gathered}
$$

The Kondo temperature in this case is given by ${ }^{62}$

$$
T_{K} \propto D\left(\frac{J_{\perp}}{J_{\|}}\right)^{1 / J_{\|} \rho_{0}} .
$$

In the limit of large $\lambda / \omega_{0}^{2}, J_{\perp} / J_{\|}$behave as

$$
J_{\perp} / J_{\|} \approx \exp \left[-2\left(\lambda / \omega_{0}\right)^{2}\right] .
$$

Then, the Kondo temperature $T_{K}$ reads ${ }^{45}$ 


$$
T_{K} \propto \exp \left[-\left(\frac{\lambda}{\omega_{0}}\right)^{4} \frac{\pi \omega_{0}}{\Gamma}\right] .
$$

In contrast to the weak e-ph coupling case, the Kondo temperature decreases sharply with increasing $\lambda\left(\Gamma=2 \pi \rho_{0} V^{2}\right.$, with $\rho_{0}=\rho_{L}=\rho_{R}$ being the DOS of the conduction band electrons, we took the same for both contacts). Equations (2.22) and (2.32) are the main results of this section.

\section{NUMERICAL RENORMALIZATION GROUP APPROACH}

In order to describe both the spin Kondo effect, occurring for $2 \lambda^{2} /\left(\omega_{0} U\right)<1$, and the charge Kondo effect, occurring for $2 \lambda^{2} /\left(\omega_{0} U\right)>1$, on the same footing, we have therefore used the numerical renormalization group technique. ${ }^{63,64} \mathrm{Un}$ like other approaches, the NRG is not restricted for the values of the parameters $\left(\lambda, \omega_{0}, U, \Gamma, W, \varphi\right.$, etc $)$.

NRG method is employed to solve the full Hamiltonian. This allows us to compute the linear transport properties through the $\mathrm{AB}$ interferometer such as the linear conductance. It is known that NRG method suffers from loss of accuracy coming from the discretization of the conduction band and the truncation of high energy states. This problem is serious in our system because the Hilbert space grows exponentially with the number of conduction channels in the leads. Any careless truncation can lead to significant deterioration of the results. We thus exploit recent improvements in the NRG procedure as well as a symmetry property of the system to obtain high-quality predictions. ${ }^{65}$ At very low frequencies, the NRG schemes developed by Žitko and Pruschke in Ref. 66 and Hofstetter and Zarand in Ref. 65 yield similar results. These results are in agreement with those obtained by employing directly the Friedel-Langreth sum rule $^{45}$ valid only at zero temperature. However, for the high frequency spectrum it seems more convenient to employ the NRG code, for instance, if we were interested in the vibronic sidebands, developed in Ref. 66. Since we are interested in the linear transport at zero temperature, then we simply use the Friedel sum rule that agrees with very refined NRG schemes in the low frequency regime.

According to the standard NRG procedure, Eq. (2.7) can be rewritten as (note that the conduction band is now discrete with states denoted by the $f$ operators)

$$
\begin{aligned}
\mathcal{H}= & \mathcal{H}_{M}+\mathcal{H}_{\mathrm{e}-\mathrm{ph}}+\mathcal{H}_{\mathrm{ph}}+\sum_{\sigma} \sqrt{\frac{4 \Gamma}{\pi}}\left[\cos (\varphi / 2) d_{\sigma}^{\dagger} f_{0 e \sigma}\right. \\
& \left.+\sin (\varphi / 2) d_{\sigma}^{\dagger} f_{0 o \sigma}+\text { h.c. }\right] \\
& +\frac{1+\Lambda^{-1}}{2} \sum_{\alpha=e / o} \sum_{n=0}^{\infty} \sum_{\sigma} \Lambda^{-n / 2} \zeta_{n}\left(f_{n \alpha \sigma}^{\dagger} f_{n+1 \alpha \sigma}+\text { h.c. }\right) \\
& +\frac{2}{\pi} \sqrt{\xi} \sum_{\sigma}\left(f_{0 e \sigma}^{\dagger} f_{0 e \sigma}-f_{0 o \sigma}^{\dagger} f_{0 o \sigma}\right),
\end{aligned}
$$

with $\xi \equiv \pi^{2} W^{2} \rho_{0}^{2}$ and

$$
f_{n e \sigma}=\frac{1}{\sqrt{2}}\left(e^{i \varphi / 2} f_{n L \sigma}+e^{-i \varphi / 2} f_{n R \sigma}\right),
$$

$$
f_{n o \sigma}=\frac{-i}{\sqrt{2}}\left(-e^{i \varphi / 2} f_{n L \sigma}+e^{-i \varphi / 2} f_{n R \sigma}\right),
$$

the parity basis. Now, we apply the NRG method to solve the Hamiltonian. We define a sequence of Hamiltonians $\overline{\mathcal{H}}_{N}$

$$
\begin{aligned}
\overline{\mathcal{H}}_{N}= & \Lambda^{(N-1) / 2}\left\{\mathcal{H}_{M}+\mathcal{H}_{\mathrm{e}-\mathrm{ph}}+\mathcal{H}_{\mathrm{ph}}\right. \\
& +\sum_{\sigma} \sqrt{\frac{4 \Gamma_{\sigma}}{\pi}}\left[\cos (\varphi / 2) d_{\sigma}^{\dagger} f_{0 e \sigma}+\sin (\varphi / 2) d_{\sigma \sigma}^{\dagger} f_{0 o \sigma}+\text { h.c. }\right] \\
& +\frac{1+\Lambda^{-1}}{2} \sum_{\alpha=e / o} \sum_{n=0}^{N-1} \sum_{\sigma} \Lambda^{-n / 2} \zeta_{n}\left(f_{n \alpha \sigma}^{\dagger} f_{n+1 \alpha \sigma}+\text { h.c. }\right) \\
& \left.+\frac{2}{\pi} \sqrt{\xi} \sum_{\sigma}\left(f_{0 e \sigma}^{\dagger} f_{0 e \sigma}-f_{0 o \sigma}^{\dagger} f_{0 o \sigma}\right)\right\}
\end{aligned}
$$

that follows the recursion relation:

$$
\tilde{\mathcal{H}}_{N+1}=\sqrt{\Lambda} \tilde{\mathcal{H}}_{N}+\sum_{\alpha=e / o} \sum_{\sigma} \zeta_{n}\left(f_{N \alpha \sigma}^{\dagger} f_{N+1 \alpha \sigma}+\text { h.c. }\right)
$$

with

$$
\tilde{\mathcal{H}}_{N}=\frac{2}{1+\Lambda^{-1}} \overline{\mathcal{H}}_{N} .
$$

From consecutive diagonalizations of the Hamiltonian, we obtain the eigenvalues and eigenstates. With them, we build the molecular and phonon Green's functions that are needed for the calculation of the linear transport properties. In order to improve the quality of these Green's functions, we follow Jeon et al. ${ }^{6}$ and relate the molecule and local phonon Green's functions by using the equation-of-motion (EOM) technique. We relegate the details of such a calculation to the Appendix B.

\section{LINEAR TRANSPORT: CONDUCTANCE}

The electrical current through the left barrier is calculated through the simple relation

$$
I_{L}=-e \dot{N}_{L}=\frac{-i e}{\hbar}\left[\mathcal{H}, N_{L}\right]
$$

where $N_{L}=\sum_{k \sigma} c_{L k \sigma}^{\dagger} c_{L k \sigma}$. After some algebra, the current through the $\mathrm{AB}$ interferometer takes the usual LandauerBüttiker form ${ }^{50,51}$

$$
I=\frac{e}{h} \sum_{\sigma} \int d \epsilon \mathcal{T}_{\sigma}(\epsilon)\left[f_{L}(\epsilon)-f_{R}(\epsilon)\right],
$$

where $f_{L(R)}$ is the left (right) Fermi function and the transmission probability reads

$$
\begin{aligned}
\mathcal{T}_{\sigma}(\epsilon)= & \mathcal{T}_{b}+\sqrt{\alpha \mathcal{T}_{b} \mathcal{R}_{b}} \cos (\varphi) \tilde{\Gamma} \Re\left[\mathcal{G}_{d \sigma, d \sigma}^{r}(\epsilon)\right]-\frac{1}{2}\{\alpha[1 \\
& \left.\left.-\mathcal{T}_{b} \cos ^{2}(\varphi)\right]-\mathcal{T}_{b}\right\} \widetilde{\Gamma} \Im\left[\mathcal{G}_{d \sigma, d \sigma}^{r}(\epsilon)\right],
\end{aligned}
$$

where, 


$$
\mathcal{T}_{b}=\frac{4 \xi}{(1+\xi)^{2}}
$$

is the background transmission with $\xi=\pi^{2} W^{2} \rho_{0}^{2}$. The symbols $\mathfrak{I}$ and $\mathfrak{R}$ denote the imaginary and real part of a complex number and $\mathcal{G}_{d \sigma, d \sigma}^{r}$ is the quasiparticle retarded Green's function for the localized electrons at the molecule site. The background reflection is obtained immediately by $\mathcal{R}_{b}=1$ $-\mathcal{T}_{b}$. It is also useful to define $\widetilde{\Gamma}=\Gamma /(1+\xi), \alpha=4 \Gamma_{L} \Gamma_{R} / \Gamma^{2}$ with $\Gamma=\Gamma_{L}+\Gamma_{R}\left(\Gamma_{L(R)}=\pi \rho_{L(R)} V_{L(R)}^{2}\right.$, below, we discuss only results for the case of symmetric tunneling rates, i.e., $\Gamma_{L}$ $=\Gamma_{R}=\Gamma=\pi \rho_{0} V^{2}$ with $\left.\alpha=1\right)$. In the linear response regime, Eq. (4.2) can be written as

$$
I=\lim _{V \rightarrow 0} \frac{e^{2} V}{h} \sum_{\sigma} \int d \epsilon \mathcal{T}_{\sigma}(\epsilon) f_{\mathrm{eq}}^{\prime}(\epsilon),
$$

where $f_{\text {eq }}$ is the equilibrium Fermi function. At $k_{B} T=0$,

$$
I=-\lim _{V \rightarrow 0} \frac{e^{2} V}{h} \sum_{\sigma} \int d \epsilon \mathcal{T}_{\sigma}(\epsilon) \delta\left(E_{F}-\epsilon\right) .
$$

Thus,

$$
G=\frac{e^{2}}{h} \sum_{\sigma} \mathcal{T}_{\sigma}\left(E_{F}\right), \quad \text { at } k_{B} T=0 .
$$

This can be further simplified by taking the quasiparticle Green's function for the localized electrons written in a Dyson type equation as follows:

$$
\mathcal{G}_{d \sigma, d \sigma}^{r}(\epsilon)=\frac{1}{\epsilon-\varepsilon_{d}-\Sigma(\epsilon)} .
$$

At $k_{B} T=0$, in the pure Kondo regime, the imaginary part of the interaction self-energy evaluated at the Fermi energy vanishes, then we have

$$
\mathfrak{I}\left[\Sigma\left(E_{F}\right)\right]=-\frac{\tilde{\Gamma}}{2}, \quad q=\frac{2}{\widetilde{\Gamma}}\left\{\varepsilon_{d}+\mathfrak{R}\left[\Sigma\left(E_{F}\right)\right]\right\} .
$$

Using this notation, we can write a more transparent expression for the transmission probability in a generalized Fano form as

$$
\mathcal{T}\left(E_{F}\right)=\mathcal{T}_{b} \frac{(q+\mathcal{F})^{2}}{e^{2}+1}+\alpha \frac{\sin ^{2}(\varphi)}{q^{2}+1},
$$

with a Fano parameter given by

$$
\mathcal{F}=-\cot \left(\delta_{\text {non }}\right)=-\sqrt{\frac{\alpha \mathcal{R}_{b}}{\mathcal{T}_{b}}} \cos (\varphi) .
$$

Notice that we have introduced $\delta_{\text {non }}$ as a nonresonant phase shift owing to scattering of electrons through the direct path. Alternatively, in terms of resonant and nonresonant phase shifts, the transmission probability can be written as:

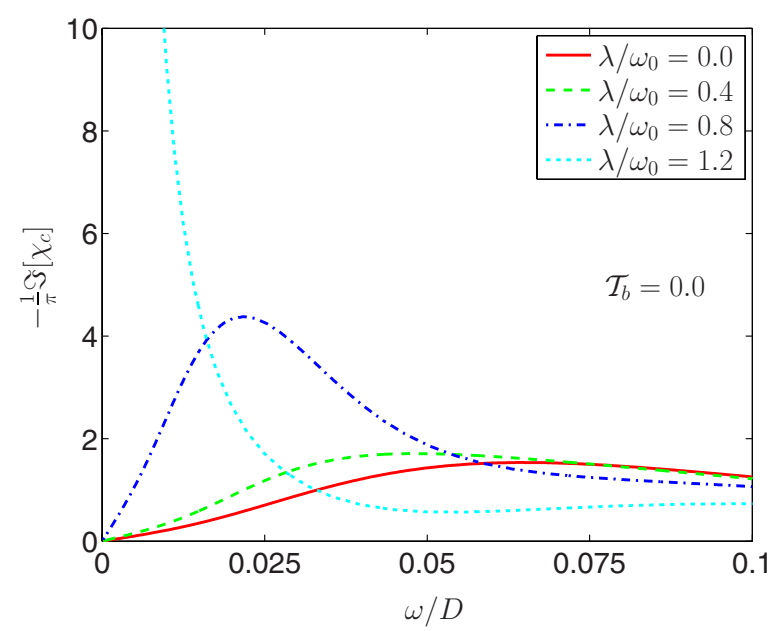

FIG. 2. (Color online) Charge susceptibility $\chi_{c}(\omega)$ as a function of the e-ph coupling $\lambda / \omega_{0}$. Parameters are $\varepsilon_{d}=0.05, U=0.1,2 \Gamma$ $=0.0016, \varphi=0, \mathcal{T}_{b}=0, k_{B} T=0$.

$$
\begin{aligned}
\mathcal{T}\left(E_{F}\right)= & {\left[\mathcal{T}_{b}+\alpha \mathcal{R}_{b} \cos ^{2}(\varphi)\right] \sin ^{2}\left(\delta_{\text {res }}-\delta_{\text {non }}\right) } \\
& +\alpha \sin ^{2}(\varphi) \sin ^{2}\left(\delta_{\text {res }}\right)=\mathcal{T}_{b} \frac{\sin ^{2}\left(\delta_{\text {res }}-\delta_{\text {non }}\right)}{\sin ^{2}\left(\delta_{\text {non }}\right)} \\
& +\alpha \sin ^{2}(\varphi) \sin ^{2}\left(\delta_{\text {res }}\right),
\end{aligned}
$$

where the resonant scattering phase shift $\delta_{\text {res }}$ can be related to the quantum average occupation number $n_{d}=\Sigma_{\sigma}\left\langle d_{\sigma}^{\dagger} d_{\sigma}\right\rangle$ using the Friedel-Langreth sum rule ${ }^{45}$

$$
\delta_{\mathrm{res}}=\frac{\pi n_{d}}{2}=\tan ^{-1}\left\{\frac{\tilde{\Gamma}}{\varepsilon_{d}+\mathfrak{R}\left[\Sigma\left(E_{F}\right)\right]}\right\} .
$$

\section{NUMERICAL RESULTS}

In the following, we present our numerical results based on the NRG method. We fix $U=0.1,2 \Gamma=0.016, \omega_{0}=0.05$, and $k_{B} T=0$ and we vary the rest of parameters. We have chosen a value of the phonon vibrational frequency $\omega_{0}$ on the order of the Coulomb energy scale, which corresponds with the experimental situation. Using these parameters, the bipolaronic attraction occurs for $\lambda / \omega_{0}>1$, whereas the weak e-ph coupling limit takes place for $\lambda / \omega_{0}<1$.

We start investigating how are affected the Kondo correlations by the presence of phonons $(\lambda \neq 0)$. In order to do, so we expand Eq. (4.12) around the symmetric point, $\varepsilon_{d}^{\star}=$ $-U / 2$ (for $\mathcal{T}_{b}=0$ and $\varphi=0$ ), we have

$$
G\left(\varepsilon_{d}\right) \approx G\left(\varepsilon_{d}^{\star}\right)-G_{0}\left(\frac{\pi \chi_{c}}{2}\right)^{2}\left(\varepsilon_{d}-\varepsilon_{d}^{\star}\right)^{2},
$$

where, $\quad \chi_{c}=\left\langle\left\langle n_{d}(t), n_{d}(0)\right\rangle\right\rangle=-i \Theta(t)\left\langle\left[n_{d}(t), n_{d}(0)\right]_{-}\right\rangle$denotes the charge susceptibility. Figure 2 shows the charge susceptibility $\chi_{c}$ as a function of the e-ph coupling $\lambda / \omega_{0}$. We see that the charge susceptibility presents a wide peak for $\lambda=0$ (almost flat charge susceptibility spectrum). Then, the broad peak decreases and moves toward higher frequencies whereas a low energy peak emerges in the spectrum as long 


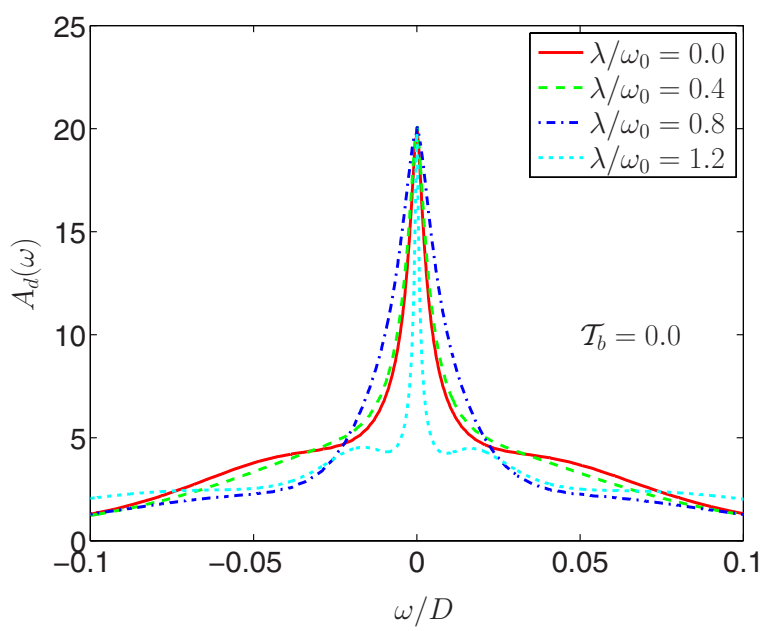

FIG. 3. (Color online) Electronic spectral density $A_{d}(\omega)$ for different values of $\lambda / \omega_{0}$ and $\mathcal{T}_{b}=0$. Parameters are $\varepsilon_{d}=-0.05, U$ $=0.1,2 \Gamma=0.016, \varphi=0$, and $k_{B} T=0$.

as $\lambda / \omega_{0}$ grows. The low energy peak corresponds to charge Kondo correlations that have a different energy scale $\left(T_{K}\right)$ than the spin Kondo correlations (corresponding to the broad peak). This result demonstrates that the e-ph coupling induces Kondo correlations in the charge sector reflected in the appearance of a low-energy peak in $\chi_{c}$.

In the following, we analyze the local density of states of the molecule for $\varphi=0$ both in the weak and strong e-ph coupling. We start by showing in Fig. 3 the DOS in the absence of the direct channel, i.e., $W=0$ for the symmetric case $\varepsilon_{d}$ $=-U / 2$. Here, the main effect of the e-ph interaction is to broad the Kondo resonance when $\lambda / \omega_{0}<1$ and to shrink it when $\lambda / \omega_{0}>1$ (see, for example, the curve corresponding to $\lambda / \omega_{0}=1.2$, which corresponds to the strong e-ph coupling regime). In this case, $T_{K}$ becomes very small as expected from Eq. (2.32). When the direct channel is connected, we see in Fig. 4 that the Kondo resonance is almost unaffected in the weak e-ph coupling regime $\left(\lambda / \omega_{0}<1\right)$. However,

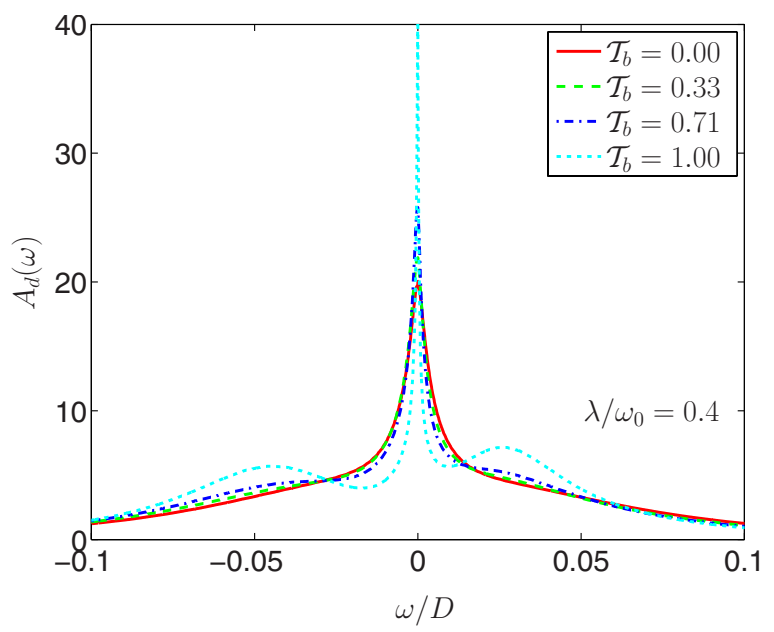

FIG. 4. (Color online) Electronic spectral density $A_{d}(\omega)$ for $\lambda / \omega_{0}=0.4$ and different values of $\mathcal{T}_{b}$. This corresponds to the weak e-ph coupling regime. Parameters are $\varepsilon_{d}=-0.05, U=0.1,2 \Gamma$ $=0.016, \varphi=0$, and $k_{B} T=0$.

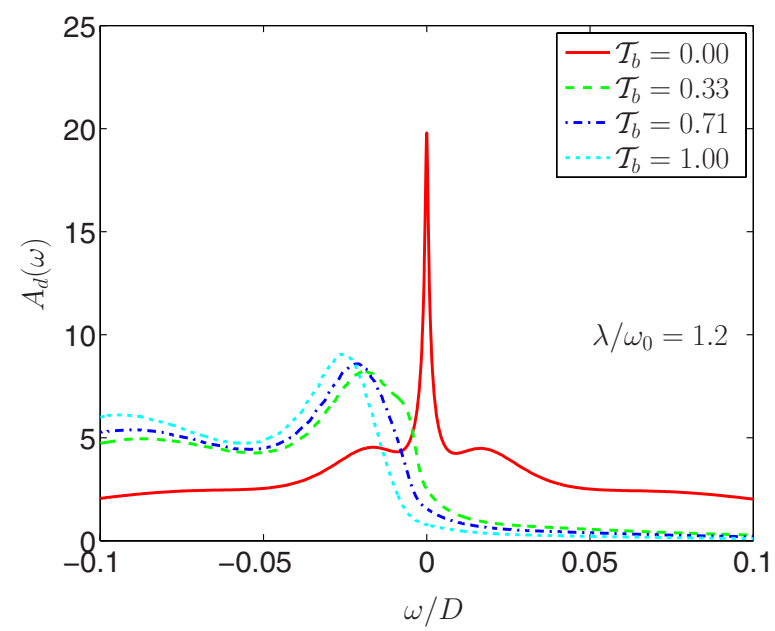

FIG. 5. (Color online) Electronic spectral density $A_{d}(\omega)$ for $\lambda / \omega_{0}=1.2$ and different values of $\mathcal{T}_{b}$. This corresponds to the strong e-ph coupling regime. Parameters are $\varepsilon_{d}=-0.05, U=0.1,2 \Gamma$ $=0.016, \varphi=0$, and $k_{B} T=0$.

there is a remarkable feature, the DOS becomes asymmetric. The asymmetry in the DOS indicates clearly the electronhole (e-h) symmetry breaking. This lack of e-h symmetry has dramatic consequences in the strong e-ph coupling regime where the Kondo effect is due to charge fluctuations. Figure 5 shows the behavior of the DOS when $\mathcal{T}_{b} \neq 0$. For $\mathcal{T}_{b}=0$, we see that the charge Kondo resonance is pinned at $E_{F}$ due to the particle-hole symmetry (remember that $\left.\varepsilon_{d}=-U / 2\right)$. As long as $\mathcal{T}_{b}$ is turned on, the e-h symmetry is broken and the charge Kondo effect is rapidly destroyed.

All these features are displayed in the linear conductance. Here, we have analyzed the linear conductance for different e-ph couplings, different background transmission $\mathcal{T}_{b}$, and Aharonov-Bohm phases. Figure 6 displays the zero temperature linear conductance $\mathcal{G}$ as a function of the level position $\varepsilon_{d}$ in the absence of the e-ph coupling, i.e., $\lambda=0$. Different curves correspond to different values of background transmission $\mathcal{T}_{b}$. For $\mathcal{T}_{b}=0$, the conductance reaches the unitary

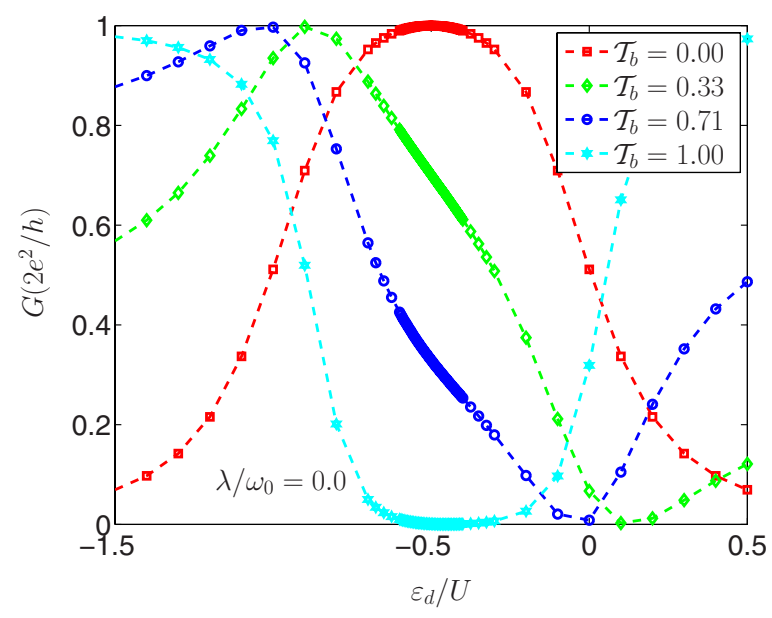

FIG. 6. (Color online) Linear conductance $\mathcal{G}$ as a function of a gate voltage $\varepsilon_{d}$ for different values of background transmission $\mathcal{T}_{b}$ at $k_{B} T=0$. Parameters are $U=0.1,2 \Gamma=0.016, \lambda=0$, and $\varphi=0$. 
limit, i.e., $\mathcal{G}=2 e^{2} / h$ in the Kondo regime $\left(-U+\Gamma<\varepsilon_{d}<\right.$ $-\Gamma)$ and then it drops rapidly when charge fluctuations are turned on, i.e., whenever $-\Gamma<\varepsilon_{d}<\Gamma$ and $-U-\Gamma<\varepsilon_{d}<-U$ $+\Gamma$. Finally, in the empty orbital regime the molecule charge $n_{d} \approx 0,2$ leading to a vanishing conductance. This scenario is totally modified when the direct path is connected. In such a case, interference effects between the resonant and nonresonant paths give rise to asymmetric line-shape for the conductance and eventually a zero conductance region for $\mathcal{T}_{b}=1$ in the Kondo regime, due to the destructive interference. There is, however, a tiny feature in the linear conductance not noticed so far due to the lack of e-h symmetry. The Kondo plateau (for $\mathcal{T}_{b}=1$ ) is not totally symmetric with respect to $\varepsilon_{d} / U=-0.5$ as it should be in the case of e-h symmetry. This is because the direct path breaks this symmetry. However, this has a negligible impact when the Kondo correlations are generated by spin fluctuations as we have shown. However, the lack of e-h symmetry has stronger consequences on the conductance in the strong e-ph coupling corresponding to the charge Kondo effect.

Now, let us discuss the conductance behavior with $\mathcal{T}_{b}$ in terms of resonant and nonresonant phase shifts. In the Kondo regime $(\lambda=0)$, we can safely assume that the resonant phase shift $\delta_{\text {res }}=\pi / 2$. Taking this fact into account, Eq. (4.12) is greatly simplified

$$
\mathcal{T}\left(E_{F}\right)\left[\delta_{\text {res }}=\pi / 2\right]=\alpha\left[1-\mathcal{T}_{b} \cos ^{2}(\varphi)\right],
$$

Therefore the linear conductance decreases as long as we open the direct path by increasing $\mathcal{T}_{b}$. It is important to realize that Kondo correlations are not destroyed in spite of the fact that linear conductance is suppressed. The reduction in the conductance is rather due to interference between the lower and the upper paths. Notice that here the conductance reduction is maintained for any value of $\varepsilon_{d}$ in the Kondo regime $\left(-U+\Gamma<\varepsilon_{d}<-\Gamma\right)$, whereas for a noninteracting quantum dot this is only true for the resonant condition when the resonant level matches with the Fermi energy in which the phase is $\delta_{\text {res }}=\pi / 2$. Finally, away from the Kondo region $\delta_{\text {res }} \rightarrow 0, \pi$, and then $\mathcal{T}\left(E_{F}\right)$ is equivalent to the nonresonant transmission probability $\mathcal{T}_{b}$. In general for arbitrary $\delta_{\text {res }}$ and $\varphi=0$, we obtain

$$
\mathcal{T}\left(E_{F}\right)=\left(\mathcal{T}_{b}+\alpha \mathcal{R}_{b}\right) \sin ^{2}\left(\delta_{\text {res }}-\delta_{\text {non }}\right) .
$$

This expression leads to a conductance peak whenever $\delta_{\text {res }}$ $=\delta_{\text {non }} \pm \pi / 2$ and a vanishing conductance for $\delta_{\text {res }}=\delta_{\text {non }}$. For $\mathcal{T}_{b}=0$, in the Kondo region, the conductance reaches its unitary value $\left(\delta_{\text {res }}=\pi / 2\right.$ and $\left.\delta_{\text {non }}=0\right)$ and vanishes outside $\left(\delta_{\text {res }}=\delta_{\text {non }}=0\right)$. Now, we turn on $\mathcal{T}_{b}$ and the linear conductance is gradually suppressed in the Kondo regime when $\mathcal{T}_{b}$ is augmented $\left[\delta_{\text {res }}=\pi / 2\right.$ and $\cot \left(\delta_{\text {non }}\right)=\sqrt{\alpha \mathcal{R}_{b} / \mathcal{T}_{b}}$, i.e., $\delta_{\text {non }}$ $\rightarrow 0$ for $\mathcal{T}_{b} \rightarrow 0$ and $\delta_{\text {non }} \rightarrow \pi / 2$ for $\left.\mathcal{T}_{b} \rightarrow 1\right]$. Outside the Kondo regime, $\mathcal{G}$ increases ( $\delta_{\text {res }}=0$ and $\delta_{\text {non }} \neq 0$ ). Eventually, for $\mathcal{T}_{b}=1, \mathcal{G}$ vanishes in the Kondo regime $\left(\delta_{\text {non,res }}=\pi / 2\right)$. Away from this regime, the conductance presents a peak since $\delta_{\text {non }}=\pi / 2$ and $\delta_{\text {res }}=0$.

This physical scenario is modified when $\lambda \neq 0$ as shown in Fig. 7. The main difference with the $\lambda=0$ case (cf. Figure 6 ) is that the conductance width decreases due to the presence of phonons. This conductance width is roughly given by

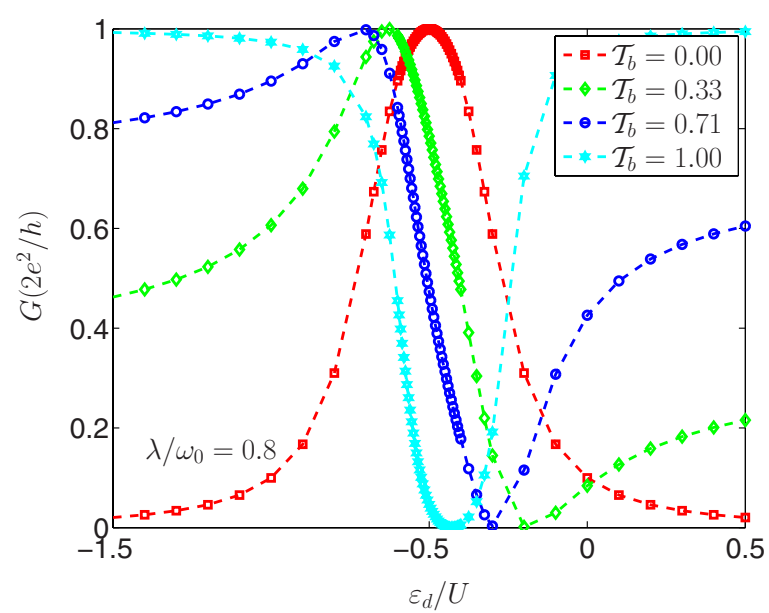

FIG. 7. (Color online) Linear conductance $\mathcal{G}$ as a function of a gate voltage $\varepsilon_{d}$ for different values of background transmission $\mathcal{T}_{b}$ at $k_{B} T=0$. Parameters are $U=0.1,2 \Gamma=0.016, \lambda / \omega_{0}=0.8, \omega_{0}=0.05$, and $\varphi=0$.

the e-e repulsion, since $U$ is effectively renormalized by the electron-phonon interaction $\left(U_{\text {eff }}=U-2 \lambda^{2} / \omega_{0}\right)$ the Kondo plateau region is reduced as long as $\lambda / \omega_{0}$ increases. A more striking feature observed in the linear conductance is the evident lack of e-h symmetry when $\mathcal{T}_{b} \neq 0$. In Fig. 6 when $\mathcal{T}_{b}$ $=1$ the conductance curve is not symmetric with respect to $\varepsilon_{d} / U=-0.5$ reflecting the lack of e-h symmetry. The effect of the e-h symmetry breaking is more pronounced as long as $\lambda / \omega_{0}$ grows and the system enters in the charge Kondo effect originated by the bipolaronic attraction. In this regime, the lack of e-h symmetry for $\varepsilon_{d} / U=-0.5$ results in a complete suppression of the charge Kondo effect $\left(\lambda / \omega_{0}>1\right)$ with strong consequences in the linear conductance. The linear conductance for the bipolaron regime (strong e-ph coupling limit) is plotted in Fig. 8. In the absence of the direct path the conductance consists of a very narrow peak of unitary conductance around $\varepsilon_{d} / U=-0.5$. The width of such a peak reflects the fact that the charge Kondo effect occurs only for
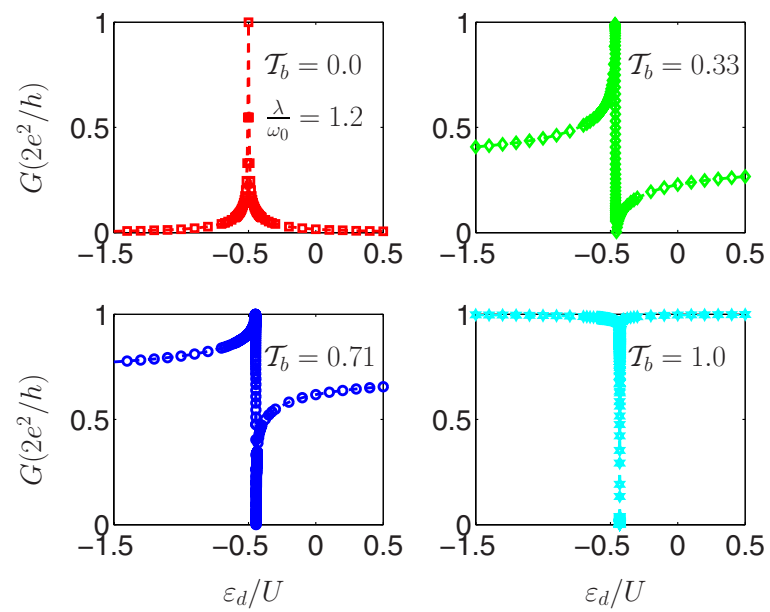

FIG. 8. (Color online) Linear conductance $\mathcal{G}$ versus gate voltage $\varepsilon_{d}$ for different values of $\mathcal{T}_{b}$ at $k_{B} T=0$ in the strong e-ph coupling regime, $\lambda / \omega_{0}=1.2$. Parameters are $U=0.1,2 \Gamma=0.016, \omega_{0}=0.05$, and $\varphi=0$. 


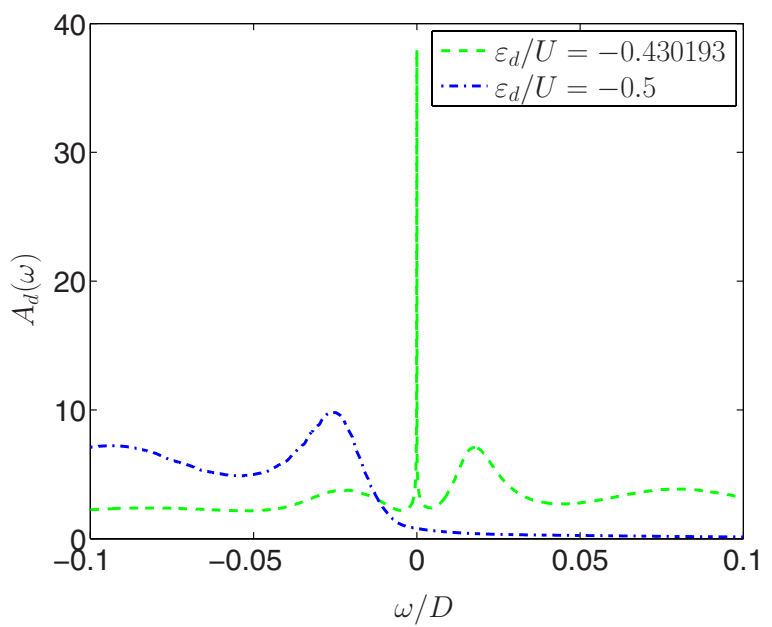

FIG. 9. (Color online) Electronic spectral density $A_{d}(\omega)$ at two different values of $\varepsilon_{d}$ with $\mathcal{T}_{b}=1$. Parameters are $\varepsilon_{d}=-0.05, U$ $=0.1,2 \Gamma=0.016, \varphi=0$, and $k_{B} T=0$.

$\Delta \approx T_{K}$ (with $T_{K}$ being the charge Kondo temperature). Any deviation from the e-h symmetry point makes the conductance drops very rapidly to zero. To turn on $\mathcal{T}_{b}$ has two different consequences. First, the direct path breaks the e-h symmetry and therefore the charge Kondo effect. However, by tuning properly $\varepsilon_{d} / U$, the Kondo resonance can be restored. Second, once the Kondo resonance is restored at certain value of $\varepsilon_{d} / U$, the interference between the Kondo resonance and the direct path can occur. These two effects are nicely illustrated in Figs. 8 and 9. Let us consider the linear conductance for $\mathcal{T}_{b}=0.33$ (dot-dashed curve in Fig. 8). Here, $\mathcal{G}$ shows a Fano-like shape where the maximal and minimal conductance take place for a value of $\varepsilon_{d} / U$ slightly above the symmetric case $\left(\varepsilon_{d} / U=-0.5\right)$. Around this value, the DOS displays a charge Kondo resonance (not shown here). Even more evident is the effect of the direct path when $\mathcal{T}_{b}=1$. As shown in Fig. 8, the complete suppression of the linear conductance occurs for $\varepsilon_{d} / U=-0.43$ since the Kondo restoration takes place at this gate value. The restoration of the Kondo resonance is showed in Fig. 9. Here, the DOS displays a Kondo resonance at $E_{F}$. Nevertheless, the restoration of the Kondo effect does not imply the restoration of the e-h symmetry since the DOS for $\varepsilon_{d} / U=-0.43$ is asymmetric (see in Fig. 9).

Finally, we comment the results for the linear conductance as a function of the $\mathrm{AB}$ phase for the two regimes. In Figs. 10 and 11 , we display the linear conductance $\mathcal{G}$ as a function of the AB phase $\varphi$. We have considered only the symmetric case, $\varepsilon_{d}=-U / 2$. From Eq. (4.12), we note that $\mathcal{G}(\varphi)=\mathcal{G}($ $-\varphi$ ). Thus, we plot only $\varphi \in[0, \pi]$. As we mentioned, the direct path breaks the e-h symmetry. This is true for any value of $\varphi$ except for the special case of $\varphi=\pi / 2$. For $\varphi$ $=\pi / 2$, the charge Kondo state is not destroyed by the direct channel and the linear conductance is given by means of Eq. (5.2). This is clearer if we use the parity basis where the odd and even channels are equally coupled to the molecule and the e-h symmetry remains unaltered independently of $\mathcal{T}_{b}$. To illustrate this we have plotted in Fig. 12 the spectral density at $\varphi=\pi / 2$ for various values of the background transmission

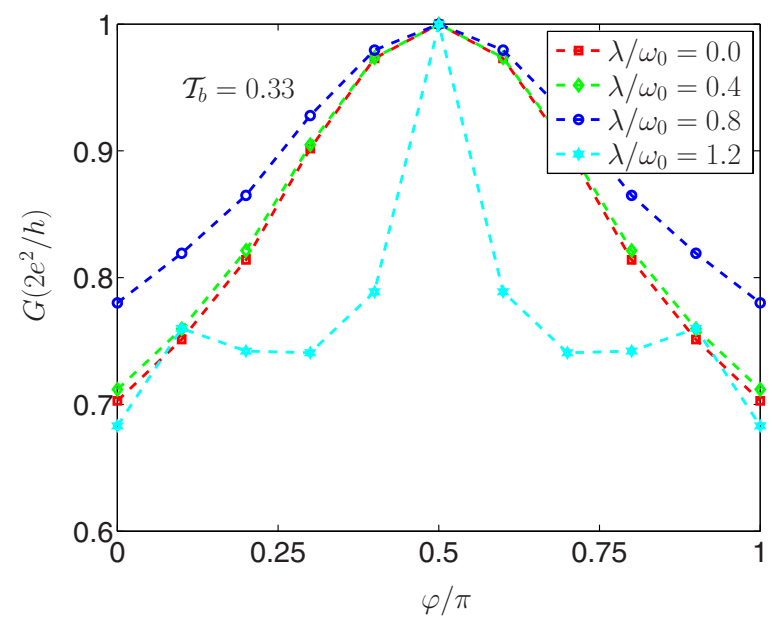

FIG. 10. (Color online) $\mathrm{AB}$ oscillations of the linear conductance $\mathcal{G}$ at $k_{B} T=0$ as a function of $\varphi$ for $\mathcal{T}_{b}=0.33$. Parameters are $\varepsilon_{d}=-0.05, U=0.1,2 \Gamma=0.016$, and $\omega_{0}=0.05$.

$\mathcal{T}_{b}$. Having this in mind, we can easily explain the results plotted in Fig. 10. In the absence of e-ph coupling, the linear conductance for the Kondo regime follows Eq. (5.2). For the weak e-ph coupling regime $\left(\lambda / \omega_{0}=0.4\right.$, and $\left.\lambda / \omega_{0}=0.8\right)$, Eq. (5.2) is still valid to describe $\mathcal{G}(\varphi)$ since for $\varepsilon_{d}=-0.5$ the system is found in the purely spin Kondo regime, only $U$ $\rightarrow U_{\text {eff }}$ and $\varepsilon_{d} \rightarrow \varepsilon_{d, \text { eff }}$. However, for $\lambda / \omega_{0}=1.2$, the system is in the strong e-ph coupling regime and the Kondo effect is partially suppressed for $\varphi \neq \pi / 2$ due to the e-h symmetry breaking. For $\varphi \approx \pi / 2$, the conductance shows a peak and only for $\varphi=\pi / 2$ the conductance is unitary due to the presence of the charge Kondo resonance and its constructive interference with the nonresonant path. For $\mathcal{T}_{b}=0.33$ and $\varphi$ $\approx 0, \pi$ the Kondo resonance is not totally quenched (see Fig. 5 for $\varphi=0$ ) and therefore the conductance is not only reduced to the background transmission but it has some contributions coming from interference between the Kondo resonant path and the direct path.

Figure 11 shows $\mathcal{G}$ versus $\varphi$ when the direct path is completely opened, i.e., $\mathcal{T}_{b}=1$. For $\lambda / \omega_{0}=0,0.4,0.8$, all these

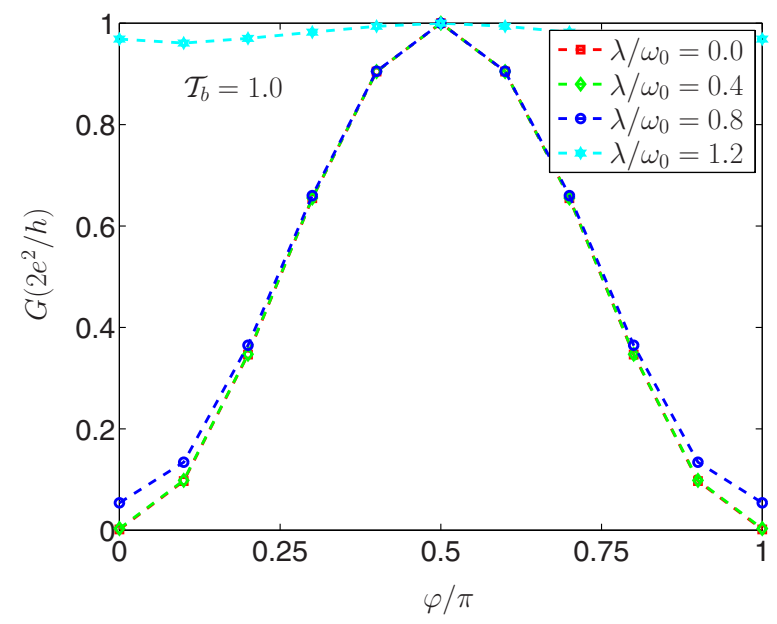

FIG. 11. (Color online) AB oscillations of the linear conductance $\mathcal{G}$ at $k_{B} T=0$ as a function of $\varphi$ for $\mathcal{T}_{b}=1$. Parameters are $\varepsilon_{d}$ $=-0.05, U=0.1,2 \Gamma=0.016$, and $\omega_{0}=0.05$. 


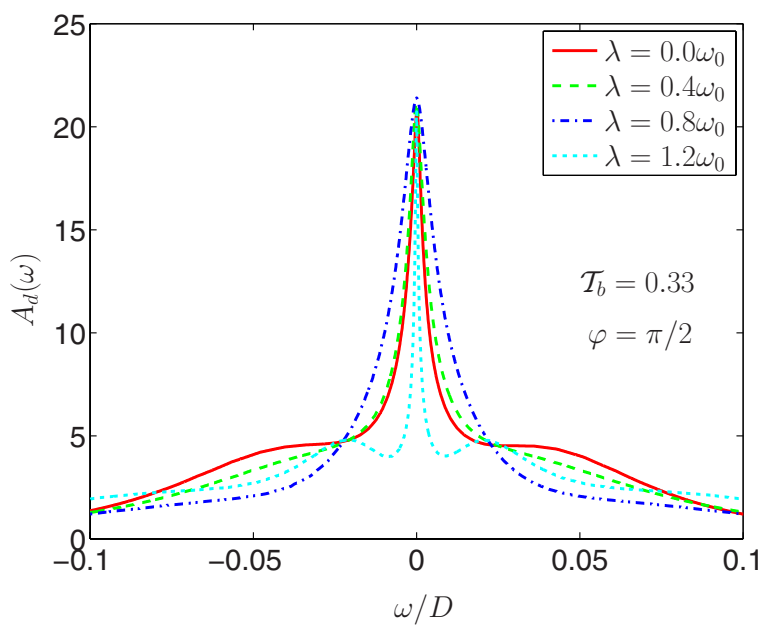

FIG. 12. (Color online) Electronic spectral density $A_{d}(\omega)$ for different values of $\lambda / \omega_{0}$ and $\mathcal{T}_{b}=0.33$. Parameters are $\varepsilon_{d}=-0.05$, $U=0.1,2 \Gamma=0.016, \varphi=\pi / 2$, and $k_{B} T=0$.

curves follows Eq. (5.2) that results from the interference between the spin Kondo resonance and the direct channel. However, for $\lambda / \omega_{0}=1.2$, the conductance shows only contribution of the background transmission probability and a tiny contribution attributed to the interference between a strong suppressed Kondo effect and the direct path that makes not unitary the conductance for $\varphi \approx \pi, 0$. At $\varphi=\pi / 2$, the conductance reaches the unitary value since for this special phase value the charge Kondo effect is not destroyed and we have constructive interference.

\section{CONCLUSIONS}

We have analyzed the linear transport properties of an $\mathrm{AB}$ interferometer with a molecule inserted in one of its arms. The vibronic internal degrees of freedom of the molecule are described by means of phonons modes coupled to the molecular occupation. Depending on the e-ph coupling, we distinguish two different scenarios, the weak e-ph coupling limit where the electronic transport occurs as single electron tunneling events and the strong e-ph interaction, which leads to bipolaronic attraction between electrons. Here, the tunneling through the molecule takes place in pair of electrons. We investigate the low temperature limit where Kondo correlations emerge when the molecule is strongly coupled to the leads. Depending on the e-ph coupling, the Kondo correlations have a different origin. For the weak e-ph coupling, the Kondo effect corresponds to the spin Kondo effect, while for a strong e-ph interaction, the bipolaron formation leads to the charge Kondo effect with fluctuations between zero and double charge states (bipolaron state). The effect of the direct path breaks the e-h symmetry with dramatic consequences in the transport properties. We show that the direct path destroys completely the charge Kondo effect and suppresses the interference between the resonant and nonresonant paths. By a proper tuning of the molecular level position, it is possible to restore the Kondo resonance but not the e-h symmetry. In this case, when the charge Kondo state is revived, interference effects come up. The usual spin Kondo effect is less sensitive to the e-h symmetry and is therefore less affected by the direct path. In this case, the transport properties in the weak e-ph coupling regime are dominated by the interferences between the spin Kondo effect and the direct path. In order to obtain these results, we have chosen values for the parameters, which are in the range of typical experiments in molecular transport and we are therefore confident that our predictions can be tested in the near future.

\section{ACKNOWLEDGMENTS}

We acknowledge D. Sánchez and R. Žitko for their fruitful discussions. P.S. would like to acknowledge O. Entin-Wohlman and A. Aharony for an earlier collaboration on related issues. R.L. and J.S.L acknowledge financial support from Grant No. FIS2008-00781 and the Conselleria d'Innovació, Interior i Justicia (before Conselleria d'Economia, Hisenda i Innovació), Govern de les Illes Baleares. G.P. acknowledges financial support from Grant No. MEC: MAT2008-02626.

\section{APPENDIX A: MAPPING TO A KONDO HAMILTONIAN}

In this appendix, we give the details for the derivation of effective Hamiltonian using the projection method. This consists of a second order Rayleigh-Schrödinger perturbative calculation on the tunneling Hamiltonian, $\mathcal{H}_{T}{ }^{45}$ We analyze the two cases, namely, the weak e-ph coupling and the strong e-ph coupling regime.

Let us take $\psi_{n}$ as the component in which the occupation number of the molecule is $n_{d}$, then we can write the following matrix derived from the Schrödinger equation:

$$
\left[\begin{array}{ccc}
E-\mathcal{H}_{00} & -\mathcal{H}_{01} & -\mathcal{H}_{02} \\
-\mathcal{H}_{10} & E-\mathcal{H}_{11} & -\mathcal{H}_{12} \\
-\mathcal{H}_{20} & -\mathcal{H}_{21} & E-\mathcal{H}_{22}
\end{array}\right]\left[\begin{array}{l}
\psi_{0} \\
\psi_{1} \\
\psi_{2}
\end{array}\right]=0,
$$

where $\mathcal{H}_{n n^{\prime}}=P_{n} \mathcal{H} P_{n^{\prime}}$. Here, $P_{n}$ are the local projectors defined as

$$
\begin{gathered}
P_{0}=|0\rangle\langle 0|=\left(1-n_{\uparrow}\right)\left(1-n_{\downarrow}\right), \\
P_{\uparrow}=|\uparrow\rangle\langle\uparrow|=n_{\uparrow}\left(1-n_{\downarrow}\right), \\
P_{\downarrow}=|\downarrow\rangle\langle\downarrow|=n_{\downarrow}\left(1-n_{\uparrow}\right), \\
P_{2}=|\uparrow \downarrow\rangle\langle\uparrow \downarrow|=n_{\uparrow} n_{\downarrow},
\end{gathered}
$$

where

$$
P_{0}+P_{\uparrow}+P_{\downarrow}+P_{2}=1 \text {. }
$$

In this fashion, the effective Hamiltonian will be given by (we only write the off-diagonal contributions)

$$
\mathcal{H}_{\text {eff }}=\mathcal{H}_{10} \frac{1}{E_{1,0}-E_{0, m}} \mathcal{H}_{01}+\mathcal{H}_{12} \frac{1}{E_{1,0}-E_{2, m}} \mathcal{H}_{21},
$$

where the energies $E_{1,0}, E_{0, m}, E_{2, m}$ are the eigenenergies of the isolated molecule coupled to the local phonon mode. 


\section{Weak electron-phonon coupling limit}

The eigenenergies of the isolated molecule and the local projectors to perform the second-order perturbative calculation are given, respectively, by

$$
\begin{gathered}
E_{1,0}=\varepsilon_{d}, \\
E_{0, m}=-\frac{\lambda^{2}}{\omega_{0}}+m \omega_{0}, \\
E_{2, m}=-\frac{\lambda^{2}}{\omega_{0}}+2 \varepsilon_{d}+U+m \omega_{0},
\end{gathered}
$$

$$
\mathcal{H}_{01}=\sqrt{2} \sum_{\ell, k, \sigma, m} V_{\ell}\left\langle m\left|\mathcal{U}^{-}\right| 0\right\rangle c_{\ell k \sigma}^{\dagger} d_{\sigma}\left(1-n_{d \bar{\sigma}}\right)
$$

$$
\mathcal{H}_{21}=\sqrt{2} \sum_{\ell, k, \sigma, m} V_{\ell}^{*}\left\langle m\left|\mathcal{U}^{+}\right| 0\right\rangle \operatorname{sgn}(\sigma) d_{\sigma}^{\dagger} n_{d \bar{\sigma}} c_{\ell k \sigma}
$$

with $\bar{\sigma}=-\sigma$. The tunneling amplitudes for the even and odd channels are, respectively, $V_{e}=\cos (\varphi / 2) \mathrm{V}$ and $V_{o}$ $=\sin (\varphi / 2) V$. Using the above expressions, the effective Hamiltonian is

$$
\begin{gathered}
\mathcal{H}_{10} \frac{1}{E_{1,0}-E_{0, m}} \mathcal{H}_{01}=2 \sum_{\alpha, p, \sigma} \sum_{\beta, q, \sigma^{\prime}} \sum_{m} \frac{V_{\alpha}^{*} V_{\beta}\left|\left\langle m\left|\mathcal{U}^{-}\right| 0\right\rangle\right|^{2}}{\frac{\lambda^{2}}{\omega_{0}}+\varepsilon_{d}-m \omega_{0}}\left(1-n_{d \bar{\sigma}}\right) d_{\sigma}^{\dagger} c_{\alpha p \sigma} c_{\beta q \sigma^{\prime}}^{\dagger} d_{\sigma^{\prime}}\left(1-n_{d \bar{\sigma}^{\prime}}\right), \\
\mathcal{H}_{12} \frac{1}{E_{1,0}-E_{2, m}} \mathcal{H}_{21}=2 \sum_{\alpha, p, \sigma} \sum_{\beta, q, \sigma^{\prime}} \sum_{m} \frac{V_{\alpha} V_{\beta}^{*}\left|\left\langle m\left|\mathcal{U}^{+}\right| 0\right\rangle\right|^{2}}{\frac{\lambda^{2}}{\omega_{0}}-\varepsilon_{d}-U-m \omega_{0}} \operatorname{sgn}(\sigma) \operatorname{sgn}\left(\sigma^{\prime}\right) c_{\alpha p \sigma^{\dagger}}^{\dagger} n_{d \bar{\sigma}} d_{\sigma^{\prime}} d_{\sigma^{\prime}}^{\dagger} n_{d \bar{\sigma}^{\prime}} c_{\beta q \sigma^{\prime}},
\end{gathered}
$$

Summing over the spin indices, all these contributions yield

$$
\begin{gathered}
-\sum_{\alpha, \beta, p, q} J_{0 \alpha \beta}\left(1-n_{d \downarrow}\right) d_{\uparrow}^{\dagger} d_{\uparrow}\left(1-n_{d \downarrow}\right) c_{\alpha p \uparrow} c_{\beta q \uparrow}^{\dagger}, \\
-\sum_{\alpha, \beta, p, q} J_{2 \alpha \beta} n_{d \downarrow} d_{\uparrow} d_{\uparrow}^{\dagger} n_{d \downarrow} c_{\alpha p \uparrow}^{\dagger} c_{\beta q \uparrow}, \quad \sigma=\uparrow, \quad \sigma^{\prime}=\uparrow, \\
-\sum_{\alpha, \beta, p, q} J_{0 \alpha \beta}\left(1-n_{d \downarrow}\right) d_{\uparrow}^{\dagger} d_{\downarrow}\left(1-n_{d \uparrow}\right) c_{\alpha p \uparrow} c_{\beta q \downarrow}^{\dagger} \\
-\sum_{\alpha, \beta, p, q} J_{2 \alpha \beta} n_{d \downarrow} d_{\uparrow} d_{\downarrow}^{\dagger} n_{d \uparrow} c_{\alpha p \uparrow}^{\dagger} c_{\beta q \downarrow}, \quad \sigma=\uparrow, \quad \sigma^{\prime}=\downarrow, \\
-\sum_{\alpha, \beta, p, q} J_{0 \alpha \beta}\left(1-n_{d \uparrow}\right) d_{\downarrow}^{\dagger} d_{\uparrow}\left(1-n_{d \downarrow}\right) c_{\alpha p \downarrow} c_{\beta q \uparrow}^{\dagger} \\
-\sum_{\alpha, \beta, p, q} J_{2 \alpha \beta} n_{d \uparrow} d_{\downarrow} d_{\uparrow}^{\dagger} n_{d \downarrow} c_{\alpha p \downarrow}^{\dagger} c_{\beta q \uparrow}, \quad \sigma=\downarrow, \quad \sigma^{\prime}=\uparrow, \\
-\sum_{\alpha, \beta, p, q} J_{0 \alpha \beta}\left(1-n_{d \uparrow}\right) d_{\downarrow}^{\dagger} d_{\downarrow}\left(1-n_{d \uparrow}\right) c_{\alpha p \downarrow} c_{\beta q \downarrow}^{\dagger} \\
-J_{d \uparrow} d_{\downarrow} d_{\downarrow}^{\dagger} n_{d \uparrow} c_{\alpha p \downarrow}^{\dagger} c_{\beta q \downarrow}, \quad \sigma=\downarrow, \quad \sigma^{\prime}=\downarrow,
\end{gathered}
$$

where we have defined the following quantities as the exchanged couplings:

$$
\begin{gathered}
J_{0 \alpha \beta}=-\sum_{m} \frac{2 V_{\alpha}^{*} V_{\beta}\left|\left\langle m\left|\mathcal{U}^{-}\right| 0\right\rangle\right|^{2}}{\frac{\lambda^{2}}{\omega_{0}}+\varepsilon_{d}-m \omega_{0}}, \\
J_{2 \alpha \beta}=-\sum_{m} \frac{2 V_{\alpha} V_{\beta}^{*}\left|\left\langle m\left|\mathcal{U}^{+}\right| 0\right\rangle\right|^{2}}{\frac{\lambda^{2}}{\omega_{0}}-\varepsilon_{d}-U-m \omega_{0}} .
\end{gathered}
$$

$$
\begin{aligned}
\mathcal{H}_{\mathrm{eff}}= & \sum_{\alpha, \beta, p, q} J_{0 \alpha \beta}\left[n_{d \uparrow}\left(1-n_{d \downarrow}\right)\left(1-\delta_{\alpha, \beta} \delta_{p, q}\right) c_{\beta q \uparrow}^{\dagger} c_{\alpha p \uparrow}\right. \\
& +d_{\uparrow}^{\dagger} d_{\downarrow} c_{\beta q \downarrow}^{\dagger} c_{\alpha p \uparrow}+d_{\downarrow}^{\dagger} d_{\uparrow} c_{\beta q \uparrow}^{\dagger} c_{\alpha p \downarrow}+n_{d \downarrow}\left(1-n_{d \uparrow}\right)(1 \\
& \left.\left.-\delta_{\alpha, \beta} \delta_{p, q}\right) c_{\beta q \downarrow}^{\dagger} c_{\alpha p \downarrow}\right]+\sum_{\alpha, \beta, p, q} J_{2 \alpha \beta}\left[\left(n_{d \uparrow}-1\right) n_{d \downarrow} c_{\alpha p \uparrow}^{\dagger} c_{\beta q \uparrow}\right. \\
& \left.+d_{\downarrow}^{\dagger} d_{\uparrow} c_{\alpha p \uparrow}^{\dagger} c_{\beta q \downarrow}+d_{\uparrow}^{\dagger} d_{\downarrow} c_{\alpha p \downarrow}^{\dagger} c_{\beta q \uparrow}+\left(n_{\downarrow}-1\right) n_{d \uparrow} c_{\alpha p \downarrow}^{\dagger} c_{\beta q \downarrow}\right] .
\end{aligned}
$$

Since the possibility of $p=q$ is extremely small, we can 
safely drop the Kronecker delta terms. By employing the following identities:

$$
\begin{gathered}
S^{+}=d_{\uparrow}^{\dagger} d_{\downarrow}, \quad S^{-}=d_{\downarrow}^{\dagger} d_{\uparrow}, \\
S^{z}=\frac{1}{2}\left(d_{\uparrow}^{\dagger} d_{\uparrow}-d_{\downarrow}^{\dagger} d_{\downarrow}\right)=\frac{1}{2}\left[n_{d \uparrow}\left(1-n_{d \downarrow}\right)-n_{d \downarrow}\left(1-n_{d \uparrow}\right)\right], \\
\text { Equation }(\mathrm{A} 9) \text { can be then written as } \\
\mathcal{H}_{\text {eff }}=\sum_{\alpha, \beta, p, q} J^{\alpha \beta}\left[S _ { d \uparrow } ^ { z } \cdot \left(c_{\alpha p \uparrow}^{\dagger} c_{\beta q \uparrow}-c_{\alpha p \downarrow}^{\dagger}=n_{d \uparrow}\left(1-n_{d \downarrow}\right)+n_{d \downarrow}\left(1-n_{d \uparrow}\right) . \quad(\mathrm{A} 10 c)\right.\right. \\
\left.+S^{-} \cdot c_{\alpha p \uparrow}^{\dagger} c_{\beta q \downarrow}+\frac{1}{2} \sum_{\alpha, \beta, p, q, \sigma} K^{\alpha \beta} c_{\alpha p \sigma}^{\dagger} c_{\beta q \sigma}\right] .
\end{gathered}
$$

where $J^{\alpha \beta}=\left(J_{0 \alpha \beta}+J_{2 \alpha \beta}\right)$ and $K^{\alpha \beta}=\left(J_{0 \alpha \beta}-J_{2 \alpha \beta}\right)$

\section{Strong electron-phonon coupling: The bipolaronic scenario}

Under the particle-hole transformation, Eq. (2.7) becomes

$$
\begin{gathered}
\mathcal{H}_{C}=\sum_{\ell=1 / 2, k, \sigma} \varepsilon_{k \sigma} \tilde{c}_{\ell k \sigma}^{\dagger} \tilde{c}_{\ell k \sigma}, \\
+\sum_{k, k^{\prime}, \sigma}\left[\operatorname{sgn}(\sigma) W e^{i \varphi} \widetilde{c}_{2 k^{\prime} \sigma}^{\dagger} \tilde{c}_{1 k \sigma}+\text { h.c. }\right], \\
\mathcal{H}_{M}=\sum_{\sigma} \tilde{\varepsilon}_{d \sigma} \tilde{d}_{\sigma^{\dagger}} \tilde{d}_{\sigma}+\tilde{U}_{\tilde{n}_{d \uparrow}} \tilde{n}_{d \downarrow}, \\
\mathcal{H}_{T}=\sum_{\ell=1 / 2, k, \sigma}\left(\tilde{V}_{\ell k \sigma} \tilde{c}_{\ell k \sigma}^{\dagger} \tilde{d}_{\sigma}+\text { h.c. }\right), \\
\mathcal{H}_{\mathrm{e}-\mathrm{ph}}=\lambda\left(a+a^{\dagger}\right)\left(\tilde{n}_{d \uparrow}-\tilde{n}_{d \downarrow}\right), \\
\mathcal{H}_{\mathrm{ph}}=\omega_{o} a^{\dagger} a,
\end{gathered}
$$

where $\widetilde{\varepsilon}_{d \sigma}=U / 2+\operatorname{sgn}(\sigma) \Delta / 2, \tilde{U}=-U$, and $\Delta=2 \varepsilon_{d}+U$. Assuming the symmetry condition for the conduction band $\varepsilon_{k \sigma}=-\varepsilon_{\tilde{k} \sigma}$, the tunneling amplitudes become

$$
\begin{aligned}
& \tilde{V}_{1 k \uparrow}=V_{L k \uparrow}, \quad \tilde{V}_{1 k \downarrow}=V_{R \tilde{k} \downarrow}^{*}, \\
& \tilde{V}_{2 k \uparrow}=V_{R k \uparrow}, \quad \tilde{V}_{2 k \downarrow}=V_{L \tilde{k} \downarrow}^{*} .
\end{aligned}
$$

Now we perform the Lang-Firsov transformation. ${ }^{58,59}$ The molecular part in the presence of phonons $\left(\mathcal{H}_{M}+\mathcal{H}_{\mathrm{e}-\mathrm{ph}}\right.$ $+\mathcal{H}_{\mathrm{ph}}$ ) in Eq. (A12) reads

$$
\mathcal{H}_{M}=\sum_{\sigma}\left(\widetilde{\varepsilon}_{d \sigma}-\frac{\lambda^{2}}{\omega_{0}}\right) \tilde{d}_{\sigma}^{\dagger} \tilde{d}_{\sigma}+\left(\tilde{U}+\frac{2 \lambda^{2}}{\omega_{0}}\right) \tilde{n}_{d \uparrow} \tilde{n}_{d \downarrow}+\omega_{0} a^{\dagger} a,
$$

and the tunneling part $\mathcal{H}_{T}$ is

$$
\mathcal{H}_{T}=\sum_{\ell=1 / 2, k, \sigma}\left(\tilde{V}_{\ell k \sigma} e^{-\operatorname{sgn}(\sigma) \lambda\left(a^{\dagger}-a\right) / \omega_{0}} \widetilde{c}_{\ell k \sigma}^{\dagger} \tilde{d}_{\sigma}+\text { h.c. }\right) .
$$

Next, we perform a canonical transformation of the total Hamiltonian $\left(\tilde{\mathcal{H}} \rightarrow \mathcal{O}^{\dagger} \mathcal{H O}\right)$ using the following unitary operator:

$$
\mathcal{O}=e^{\lambda / \omega_{0}\left(a^{\dagger}-a\right)\left(\tilde{n}_{d \uparrow}-\tilde{n}_{d \downarrow}\right)} .
$$

This transformation yields the following eigenstates and eigenvalues for the isolated molecule (in the equivalent model):

$$
\begin{aligned}
& |\tilde{0}, m\rangle=|\tilde{0}\rangle|m\rangle, \quad E_{0, m}^{\tilde{n}}=\frac{\lambda^{2}}{\omega_{0}}+m \omega_{0}, \\
& |\widetilde{\sigma}, m\rangle=\mathcal{U}^{\operatorname{sgn}(\widetilde{\sigma})}|\widetilde{\sigma}\rangle|m\rangle, \quad E_{\widetilde{\sigma}, m}=\widetilde{\varepsilon}_{d \sigma}+m \omega_{0}, \\
& |\tilde{2}, m\rangle=|\tilde{2}\rangle|m\rangle, \quad E_{2, m}^{\tilde{\omega}}=\frac{\lambda^{2}}{\omega_{0}}+\widetilde{\varepsilon}_{d \sigma}+\widetilde{\varepsilon}_{d \bar{\sigma}}+\tilde{U}+m \omega_{0},
\end{aligned}
$$

with

$$
\mathcal{U}^{\operatorname{sgn}(\widetilde{\sigma})}=\exp \left[\operatorname{sgn}(\widetilde{\sigma}) \frac{\lambda}{\omega_{0}}\left(a^{\dagger}-a\right)\right] .
$$

This defines the projectors for the strong e-ph coupling regime as

$$
\begin{aligned}
& \mathcal{H}_{01}=\sqrt{2} \sum_{\ell, k, \sigma, m} \tilde{V}_{\ell k \sigma}\left\langle m\left|\mathcal{U}^{-\operatorname{sgn}(\sigma)}\right| 0\right\rangle \tilde{c}_{\ell k \sigma}^{\dagger} \tilde{d}_{\sigma}\left(1-\tilde{n}_{d \bar{\sigma}}\right), \\
& \mathcal{H}_{21}=\sqrt{2} \sum_{\ell, k, \sigma, m} \tilde{V}_{\ell k \sigma}^{*}\left\langle m\left|\mathcal{U}^{+\operatorname{sgn}(\sigma)}\right| 0\right\rangle \operatorname{sgn}(\sigma) \tilde{d}_{\sigma}^{\dagger} \tilde{n}_{d \bar{\sigma}} \tilde{c}_{\ell k \sigma},
\end{aligned}
$$

with $\bar{\sigma}=-\sigma$, and $\tilde{V}_{e k \sigma}=\cos (\varphi / 2) \tilde{V}_{k \sigma}$ and $\tilde{V}_{o k \sigma}=\sin (\varphi / 2) \tilde{V}_{k \sigma}$. With these ingredients, the effective Hamiltonian is given by the sum of the following terms: 


$$
\begin{aligned}
& \mathcal{H}_{10} \frac{1}{E_{\sigma, 0}-E_{0, m}} \mathcal{H}_{01}=2 \sum_{\alpha, p, \sigma} \sum_{\beta, q, \sigma^{\prime}} \sum_{m} \frac{\widetilde{V}_{\alpha p \sigma}^{*} \widetilde{V}_{\beta q \sigma^{\prime}}\left\langle 0\left|\mathcal{U}^{\operatorname{sgn}(\sigma)}\right| m\right\rangle\left\langle m\left|\mathcal{U}^{-\operatorname{sgn}\left(\sigma^{\prime}\right)}\right| 0\right\rangle}{\left(E_{\sigma, 0}+E_{\sigma^{\prime}, 0}\right) / 2-\frac{\lambda^{2}}{\omega_{0}}-m \omega_{0}}, \\
& \left(1-\widetilde{n}_{d \bar{\sigma}}\right) \widetilde{d}_{\sigma}^{\dagger} \widetilde{c}_{\alpha p \sigma} \widetilde{c}_{\beta q \sigma^{\prime}}^{\dagger} \widetilde{d}_{\sigma^{\prime}}^{\dagger}\left(1-\widetilde{n}_{d \bar{\sigma}^{\prime}}\right), \\
& \mathcal{H}_{12} \frac{1}{E_{\sigma, 0}-E_{2, m}} \mathcal{H}_{21}=2 \sum_{\alpha, p, \sigma} \sum_{\beta, q, \sigma^{\prime}} \sum_{m} \frac{\widetilde{V}_{\alpha p \sigma} \widetilde{V}_{\beta q \sigma^{\prime}}^{*}\left\langle 0\left|\mathcal{U}^{-\operatorname{sgn}(\sigma)}\right| m\right\rangle\left\langle m\left|\mathcal{U}^{\operatorname{sgn}\left(\sigma^{\prime}\right)}\right| 0\right\rangle}{\left(E_{\sigma, 0}+E_{\sigma^{\prime}, 0}\right) / 2-\frac{\lambda^{2}}{\omega_{0}}-\widetilde{\varepsilon}_{d \sigma}-\widetilde{\varepsilon}_{d \bar{\sigma}}-\tilde{U}-m \omega_{0}}, \\
& \operatorname{sgn}(\sigma) \operatorname{sgn}\left(\sigma^{\prime}\right) \widetilde{c}_{\alpha p \sigma}^{\dagger} \widetilde{n}_{d \bar{\sigma}} \widetilde{d}_{\sigma} \widetilde{d}_{\sigma^{\prime}}^{\dagger} \widetilde{n}_{d \bar{\sigma}^{\prime}} \widetilde{c}_{\beta q \sigma^{\prime}} .
\end{aligned}
$$

The energies $E_{\sigma, 0}$ of the initial and final states can be different. We eliminate this ambiguity by choosing the average between both energy states. Finally, a similar calculation than for the weak regime, produces the following exchange couplings:

$$
\begin{gathered}
J_{\|}^{\alpha \beta}=\frac{8}{U} \sum_{m} \frac{V_{\alpha} V_{\beta}\left|\left\langle m\left|\mathcal{U}^{-}\right| 0\right\rangle\right|^{2}}{\frac{2 \lambda^{2}}{\omega_{0} U}-1+\frac{2 m \omega_{0}}{U}}, \\
J_{\perp}^{\alpha \beta}=\frac{8}{U} \sum_{m} \frac{V_{\alpha} V_{\beta}\left\langle 0\left|\mathcal{U}^{-}\right| m\right\rangle\left\langle m\left|\mathcal{U}^{-}\right| 0\right\rangle}{\frac{2 \lambda^{2}}{\omega_{0} U}-1+\frac{2 m \omega_{0}}{U}}, \\
=\frac{8}{U} \sum_{m}(-1)^{m} \frac{V_{\alpha} V_{\beta}\left|\left\langle m\left|\mathcal{U}^{-}\right| 0\right\rangle\right|^{2}}{\frac{2 \lambda^{2}}{\omega_{0} U}-1+\frac{2 m \omega_{0}}{U}},
\end{gathered}
$$

Now, we can perform an expansion in the large $\lambda / \omega_{0}^{2}$ limit

$$
\begin{aligned}
J_{\|}^{\alpha \beta}\left[\frac{8 V_{\alpha} V_{\beta}}{U}\right]^{-1}= & \frac{e^{-\left(\lambda / \omega_{0}\right)^{2}}}{\frac{2 \lambda^{2}}{\omega_{0} U}-1}+\frac{e^{-\left(\lambda / \omega_{0}\right)^{2}}\left(\frac{\lambda}{\omega_{0}}\right)^{2}}{\frac{2 \lambda^{2}}{\omega_{0} U}-1+\frac{2 \omega_{0}}{U}}+\cdots \approx \frac{\omega_{0} U}{2 \lambda^{2}} e^{-\left(\lambda / \omega_{0}\right)^{2}}\left[1+\left(\frac{\lambda}{\omega_{0}}\right)^{2}+\frac{1}{2 !}\left(\frac{\lambda}{\omega_{0}}\right)^{4}+\cdots\right]=\frac{\omega_{0} U}{2 \lambda^{2}}, J_{\perp}^{\alpha \beta}\left[\frac{8 V_{\alpha} V_{\beta}}{U}\right]^{-1} \\
& =\frac{e^{-\left(\lambda / \omega_{0}\right)^{2}}}{\frac{2 \lambda^{2}}{\omega_{0} U}-1}-\frac{e^{-\left(\lambda / \omega_{0}\right)^{2}}\left(\frac{\lambda}{\omega_{0}}\right)^{2}}{\frac{2 \lambda^{2}}{\omega_{0} U}-1+\frac{2 \omega_{0}}{U}}+\cdots \approx \frac{\omega_{0} U}{2 \lambda^{2}} e^{-\left(\lambda / \omega_{0}\right)^{2}}\left[1-\left(\frac{\lambda}{\omega_{0}}\right)^{2}+\frac{1}{2 !}\left(\frac{\lambda}{\omega_{0}}\right)^{4}+\cdots\right]=\frac{\omega_{0} U}{2 \lambda^{2}} e^{-2\left(\lambda / \omega_{0}\right)^{2}},
\end{aligned}
$$

and finally, we note that $J_{\perp}$ is exponentially suppressed in comparison with $J_{\|}$giving a very small Kondo temperature in the strong e-ph coupling regime [see Eq. (2.30)].

\section{APPENDIX B: ELECTRONIC AND PHONON GREEN'S FUNCTIONS}

In this appendix, we calculate the electronic and phonon Green's functions in order to calculate the density of states of electrons and the linear conductance. To this end, we employ the equation-of-motion (EOM) method. ${ }^{67}$ In this manner, the equation of motion for the correlator $\langle A, B\rangle$ in the frequency domain $(z)$ is given by

$$
z\langle\langle A, B\rangle\rangle_{z}+\left\langle\left\langle[\mathcal{H}, A]_{-}, B\right\rangle\right\rangle=\left\langle[A, B]_{\zeta}\right\rangle,
$$

with $\zeta=+$ if both $A$ and $B$ are fermionic operators, while $\zeta$ $=-$ otherwise. Notice that we have defined as

$$
\langle\langle A, B\rangle\rangle_{z}=\int_{-\infty}^{\infty} d t e^{i z t}\langle\langle A, B\rangle\rangle_{t}
$$




$$
\langle\langle A, B\rangle\rangle_{t}=-i \Theta(t)\left\langle[A(t), B]_{\zeta}\right\rangle .
$$

Here, $\Theta$ denotes the step function, $\langle\cdot\rangle$ is the thermodynamic average. Next, we obtain the electron Green's function in the presence of the e-ph interaction. We derived the molecule Green's function and also the lead-molecule Green's function. For $A=d_{\sigma}$ and $A=c_{\alpha k \sigma}$ with $B=d_{\sigma}^{\dagger}$, the equation of motion for the molecule and lead-molecule Green's function are, respectively,

$$
\begin{aligned}
\left(z-\epsilon_{d}\right) \mathcal{G}_{d \sigma, d \sigma}(z)= & 1+U\left\langle\left\langle d_{\sigma} d_{\bar{\sigma}}^{\dagger} d_{\bar{\sigma}}, d_{\sigma}^{\dagger}\right\rangle\right\rangle_{z}+\lambda\left\langle\left\langle d_{\sigma}\left(a+a^{\dagger}\right), d_{\sigma}^{\dagger}\right\rangle\right\rangle_{z} \\
& +\sum_{\alpha} \sum_{k} V_{\alpha}^{*}\left\langle\left\langle c_{\alpha k \sigma}, d_{\sigma}^{\dagger}\right\rangle\right\rangle_{z},
\end{aligned}
$$

and

$$
\begin{aligned}
\left(z-\varepsilon_{k \sigma}\right)\left\langle\left\langle c_{\alpha k \sigma}, d_{\sigma}^{\dagger}\right\rangle\right\rangle_{z}= & V_{\alpha}\left\langle\left\langle d_{\sigma}, d_{\sigma}^{\dagger}\right\rangle\right\rangle_{z} \\
& +W e^{i \operatorname{sgn}(\alpha) \varphi} \sum_{q}\left\langle\left\langle c_{\bar{\alpha} q \sigma}, d_{\sigma}^{\dagger}\right\rangle\right\rangle_{z},
\end{aligned}
$$

where $\operatorname{sgn}(\alpha)=-(+)$ for $\alpha=L(R)$ and $\bar{\alpha}=R(L)$. Here, Eq. (B4) can be rewritten as

$$
\sum_{p} \mathcal{G}_{L p \sigma, d \sigma}^{r}(z)=\frac{\mathcal{G}_{d \sigma, d \sigma}^{r}(z)}{1+\xi}\left[-i \pi \rho_{L} V_{L}-\pi^{2} \rho_{L} \rho_{R} W e^{-i \varphi} V_{R}\right],
$$

$$
\sum_{q} \mathcal{G}_{R q \sigma, d \sigma}^{r}(z)=\frac{\mathcal{G}_{d \sigma, d \sigma}^{r}(z)}{1+\xi}\left[-i \pi \rho_{R} V_{R}-\pi^{2} \rho_{L} \rho_{R} W e^{+i \varphi} V_{L}\right],
$$

where $\left\langle\left\langle c_{\alpha l \sigma}, d_{\sigma}^{\dagger}\right\rangle\right\rangle_{z}=\mathcal{G}_{\alpha l \sigma, d \sigma}^{r}(z)$. Taking Eqs. (B5a), (B5b), (B3), and (B4), the molecule Green's function reads

$$
\begin{aligned}
\mathcal{G}_{d \sigma, d \sigma}^{-1}(z)= & \mathcal{G}_{d \sigma, d \sigma}^{-1}(z)-U \frac{\left\langle\left\langle d_{\sigma} d_{\bar{\sigma}}^{\dagger} d_{\bar{\sigma}}, d_{\sigma}^{\dagger}\right\rangle\right\rangle_{z}}{\mathcal{G}_{d \sigma, d \sigma}(z)} \\
& -\lambda \frac{\left\langle\left\langle d_{\sigma}\left(a+a^{\dagger}\right), d_{\sigma}^{\dagger}\right\rangle\right\rangle_{z}}{\mathcal{G}_{d \sigma, d \sigma}(z)},
\end{aligned}
$$

where

$$
\mathcal{G}_{d \sigma, d \sigma}^{-1}(z)=z-\varepsilon_{d}-\frac{1}{1+\xi}\left[-2 i \Gamma-2 \pi^{2} \rho_{0}^{2}|V|^{2} W \cos (\varphi)\right] .
$$

Finally, $\left\langle\left\langle d_{\sigma} d_{\bar{\sigma}}^{\dagger} d_{\bar{\sigma}}, d_{\sigma}^{\dagger}\right\rangle\right\rangle_{z}$ and $\left\langle\left\langle d_{\sigma}\left(a+a^{\dagger}\right), d_{\sigma}^{\dagger}\right\rangle\right\rangle_{z}$ are determined from the NRG energy spectrum. In order to do this, we write them using the spectral representation

$$
\begin{aligned}
\left\langle\left\langle d_{\sigma} d_{\bar{\sigma}}^{\dagger} d_{\bar{\sigma}}, d_{\sigma}^{\dagger}\right\rangle\right\rangle_{z}= & \frac{1}{\mathcal{Z}} \sum_{n, m}\left\langle n\left|d_{\sigma}^{\dagger}\right| m\right\rangle\left\langle m\left|d_{\sigma} d_{\bar{\sigma}}^{\dagger} d_{\bar{\sigma}}\right| n\right\rangle\left(e^{-\beta E_{n}}+e^{-\beta E_{m}}\right) \\
& \times\left[\mathcal{P} \frac{1}{z-\left(E_{n}+E_{m}\right)}-i \pi \delta\left(z-E_{n}+E_{m}\right)\right],
\end{aligned}
$$

and

$$
\begin{aligned}
\left\langle\left\langle d_{\sigma}\left(a+a^{\dagger}\right), d_{\sigma}^{\dagger}\right\rangle\right\rangle_{z} \\
=\frac{1}{\mathcal{Z}} \sum_{n, m}\left\langle n\left|d_{\sigma}^{\dagger}\right| m\right\rangle\left\langle m\left|d_{\sigma}\left(a+a^{\dagger}\right)\right| n\right\rangle\left(e^{-\beta E_{n}}+e^{\left.-\beta E_{m}\right)}\right. \\
\quad \times\left[\mathcal{P} \frac{1}{z-\left(E_{n}+E_{m}\right)}-i \pi \delta\left(z-E_{n}+E_{m}\right)\right],
\end{aligned}
$$

where $\mathcal{Z}$ is the partition function and $\mathcal{P}$ denotes the principal value in the Cauchy sense.

The phonon Green's function is calculated in the same manner as the electronic Green's function by using the EOM method. Now, $A=a$ and $B=a^{\dagger}$ and then the phonon Green's function reads

$$
\left(z-\omega_{0}\right)\left\langle\left\langle a, a^{\dagger}\right\rangle\right\rangle_{z}=1+\lambda\left\langle\left\langle n_{d}, a^{\dagger}\right\rangle\right\rangle_{z} .
$$

By dividing both sides of Eq. (B10) by $\left\langle\left\langle a, a^{\dagger}\right\rangle\right\rangle_{z}$, we get a Dyson type equation for the phonon propagator

$$
\mathcal{D}^{-1}(z)=\mathcal{D}_{0}^{-1}(z)-\lambda \frac{\left\langle\left\langle n_{d}, a^{\dagger}\right\rangle\right\rangle_{z}}{\left\langle\left\langle a, a^{\dagger}\right\rangle\right\rangle_{z}},
$$

where $\mathcal{D}(z) \equiv\left\langle\left\langle a, a^{\dagger}\right\rangle\right\rangle_{z}, \mathcal{D}_{0}^{-1}(z)=z-\omega_{0}$ and $n_{d} \equiv \Sigma_{\sigma} n_{d \sigma}-1$. The propagators $\left\langle\left\langle n_{d}, a^{\dagger}\right\rangle\right\rangle_{z}$ and $\left\langle\left\langle a, a^{\dagger}\right\rangle\right\rangle_{z}$ are obtained from the NRG spectrum. Using again the spectral representation, we have

$$
\begin{aligned}
\left\langle\left\langle a, a^{\dagger}\right\rangle\right\rangle_{z}= & -\frac{1}{\mathcal{Z}} \sum_{n, m}\left|\left\langle n\left|a^{\dagger}\right| m\right\rangle\right|^{2}\left(e^{-\beta E_{n}}-e^{\left.-\beta E_{m}\right)}\right. \\
& \times\left[\mathcal{P} \frac{1}{z-\left(E_{n}-E_{m}\right)}-i \pi \delta\left(z-\left(E_{n}-E_{m}\right)\right)\right],
\end{aligned}
$$

and

$$
\begin{aligned}
\left\langle\left\langle n_{d}, a^{\dagger}\right\rangle\right\rangle_{z}= & -\frac{1}{\mathcal{Z}} \sum_{n, m}\left\langle n\left|a^{\dagger}\right| m\right\rangle\left\langle m\left|n_{d}\right| n\right\rangle\left(e^{-\beta E_{n}}-e^{\left.-\beta E_{m}\right)}\right. \\
& \times\left[\mathcal{P} \frac{1}{z-\left(E_{n}-E_{m}\right)}-i \pi \delta\left(z-\left(E_{n}-E_{m}\right)\right)\right] .
\end{aligned}
$$

This method of calculating the phonon propagator through the EOM technique [see Eq. (B11)] is much more accurate than the direct calculation of this from the NRG spectrum [see Eq. (B12)] as it has been shown by Jeon et al., in Ref. 6. 
${ }^{1}$ M. A. Reed, C. Zhou, C. J. Muller, T. P. Burgin, and J. M. Tour, Science 278, 252 (1997).

${ }^{2}$ A. Nitzan and M. A. Ratner, Science 300, 1384 (2003).

${ }^{3}$ G. Cuniberti, G. Fagas, and K. Richter, Introducing Molecular Electronics (Springer, New York, 2005).

${ }^{4}$ H. Park, J. Park, A. K. L. Lim, E. H. Anderson, A. P. Alivisatos, and P. L. McEuen, Nature (London) 407, 57 (2000).

${ }^{5}$ W. Liang, M. P. Shores, M. Bockrath, J. R. Long, and H. Park, Nature (London) 417, 725 (2002).

${ }^{6}$ G. S. Jeon, T. H. Park, and H.-Y. Choi, Phys. Rev. B 68, 045106 (2003).

${ }^{7}$ V. Sazonova, Y. Yaish, H. Ustunel, D. Roundy, T. A. Arias, and P. L. McEuen, Nature (London) 431, 284 (2004).

${ }^{8}$ L. H. Yu and D. Natelson, Nano Lett. 4, 79 (2004).

${ }^{9}$ A. N. Pasupathy, J. Park, C. Chang, A. V. Soldatov, S. Lebedkin, R. C. Bialczak, J. E. Grose, L. A. K. Donev, J. P. Sethna, D. C. Ralph, and P. L. McEuen, Nano Lett. 5, 203 (2005).

${ }^{10}$ M. Tsukada, K. Tagami, K. Hirose, and N. Koayashi, J. Phys. Soc. Jpn. 74, 1079 (2005).

${ }^{11}$ B. J. LeRoy, S. G. Lemay, J. Kong, and C. Dekker, Nature (London) 432, 371 (2004); S. Sapmaz, P. Jarillo-Herrero, Y. M. Blanter, C. Dekker, and H. S. J. van der Zant, Phys. Rev. Lett. 96, 026801 (2006); F. Kuemmeth, S. Ilani, D. C. Ralph, and P. L. McEuen, Nature (London) 452, 448 (2008); A. K. Hüttel, M. Poot, B. Witkamp, and H. S. J. van der Zant, New J. Phys. 10, 095003 (2008); A. K. Hüttel, B. Witkamp, M. Leijnse, M. R. Wegewijs, and H. S. J. van der Zant, Phys. Rev. Lett. 102, 225501 (2009); B. Lassagne, Y. Tarakanov, J. Kinaret, D. Garcia-Sanchez, and A. Bachtold, Science 325, 1107 (2009).

${ }^{12}$ R. Leturcq, C. Stampfer, K. Inderbitzin, L. Durrer, C. Hierold, E. Mariani, M. G. Schultz, F. von Oppen, and K. Ensslin, Nat. Phys. 5, 327 (2009).

${ }^{13}$ A. N. Pasupathy, R. C. Bialczak, J. Martinek, J. E. Grose, L. A. K. Donev, P. L. McEuen, and D. C. Ralph, Science 306, 86 (2004).

${ }^{14}$ M. R. Buitelaar, W. Belzig, T. Nussbaumer, B. Babic, C. Bruder, and C. Schönenberger, Phys. Rev. Lett. 91, 057005 (2003); A. Y. Kasumov, R. Deblock, M. Kociak, B. Reulet, H. Bouchiat, I. I. Khodos, Y. B. Gorbatov, V. T. Volkov, C. Journet, and M. Burghard, Science 284, 1508 (1999); B. Reulet, A. Y. Kasumov, M. Kociak, R. Deblock, I. I. Khodos, Y. B. Gorbatov, V. T. Volkov, C. Journet, and H. Bouchiat, Phys. Rev. Lett. 85, 2829 (2000); P. Jarillo-Herrero, J. A. van Dam, and L. P. Kouwenhoven, Nature (London) 439, 953 (2006).

${ }^{15}$ S. Braig and K. Flensberg, Phys. Rev. B 68, 205324 (2003).

${ }^{16}$ K. Flensberg, Phys. Rev. B 68, 205323 (2003)

${ }^{17}$ P. S. Cornaglia, H. Ness, and D. R. Grempel, Phys. Rev. Lett. 93, 147201 (2004); P. S. Cornaglia, D. R. Grempel, and H. Ness, Phys. Rev. B 71, 075320 (2005); P. S. Cornaglia, G. Usaj, and C. A. Balseiro, ibid. 76, 241403(R) (2007).

${ }^{18}$ A. Mitra, I. Aleiner, and A. J. Millis, Phys. Rev. B 69, 245302 (2004).

${ }^{19}$ J. Koch and F. von Oppen, Phys. Rev. Lett. 94, 206804 (2005).

${ }^{20}$ J. Paaske and K. Flensberg, Phys. Rev. Lett. 94, 176801 (2005).

${ }^{21}$ L. de la Vega, A. Martin-Rodero, N. Agrait, and A. Levy Yeyati, Phys. Rev. B 73, 075428 (2006).

${ }^{22}$ M.-J. Hwang, M.-S. Choi, and R. López, Phys. Rev. B 76, 165312 (2007).

${ }^{23}$ M. Galperin, M. A. Ratner, and A. Nitzan, J. Phys.: Condens. Matter 19, 103201 (2007).
${ }^{24}$ A. Martin-Rodero, A. Levy Yeyati, F. Flores, and R. C. Monreal, Phys. Rev. B 78, 235112 (2008).

${ }^{25}$ T. Holstein, Ann. Phys. (N.Y.) 8, 343 (1959).

${ }^{26}$ C. Kraiya and D. H. Evans, J. Electroanal. Chem. 565, 29 (2004).

${ }^{27}$ A. E. Hansen, A. Kristensen, S. Pedersen, C. B. Sorensen, and P. E. Lindelof, Phys. Rev. B 64, 045327 (2001).

${ }^{28}$ G. Seelig, S. Pilgram, A. N. Jordan, and M. Büttiker, Phys. Rev. B 68, 161310(R) (2003).

${ }^{29}$ U. F. Keyser, C. Fühner, S. Borck, R. J. Haug, M. Bichler, G. Abstreiter, and W. Wegscheider, Phys. Rev. Lett. 90, 196601 (2003).

${ }^{30}$ S. Datta, Electronic Transport in Mesoscopic Systems, Cambridge Studies in Semiconductor Physics and Microelectronic Engineering (Cambridge University Press, Cambridge, United Kingdom, 1997).

${ }^{31}$ Y. Gefen, Y. Imry, and M. Ya. Azbel, Surf. Sci. 142, 203 (1984).

${ }^{32}$ M. Büttiker, Y. Imry, R. Landauer, and S. Pinhas, Phys. Rev. B 31, 6207 (1985)

${ }^{33}$ M. Büttiker, Phys. Rev. B 32, 1846 (1985).

${ }^{34}$ M. Büttiker, Phys. Rev. Lett. 68, 843 (1992).

${ }^{35}$ R. A. Webb, S. Washburn, C. P. Umbach, and R. B. Laibowitz, Phys. Rev. Lett. 54, 2696 (1985).

${ }^{36}$ A. Yacoby, M. Heiblum, D. Mahalu, and H. Shtrikman, Phys. Rev. Lett. 74, 4047 (1995).

${ }^{37}$ A. Yacoby, R. Schuster, and M. Heiblum, Phys. Rev. B 53, 9583 (1996).

${ }^{38}$ E. Buks, R. Schuster, M. Heiblum, D. Mahalu, and V. Umansky, Nature (London) 391, 871 (1998).

${ }^{39}$ D. Sprinzak, E. Buks, M. Heiblum, and H. Shtrikman, Phys. Rev. Lett. 84, 5820 (2000).

${ }^{40}$ A. W. Holleitner, C. R. Decker, H. Qin, K. Eberl, and R. H. Blick, Phys. Rev. Lett. 87, 256802 (2001) A. W. Holleitner, R. H. Blick, A. K. Hüttel, K. Eberl, and J. P. Kotthaus, Science 297, 70 (2002).

${ }^{41}$ K. Kobayashi, H. Aikawa, S. Katsumoto, and Y. Iye, Phys. Rev. Lett. 88, 256806 (2002); J. Phys. Soc. Jpn. 71, 2094 (2002).

${ }^{42}$ A. Fuhrer, S. Lüscher, T. Ihn, T. Heinzel, K. Ensslin, W. Wegscheider, and M. Bichler, Nature (London) 413, 822 (2001)

${ }^{43}$ A. Aharony, O. Entin-Wohlman, B. I. Halperin, and Y. Imry, Phys. Rev. B 66, 115311 (2002); A. Aharony, O. EntinWohlman, and Y. Imry, Phys. Rev. Lett. 90, 156802 (2003).

${ }^{44}$ Y. Ji, M. Heiblum, D. Sprinzak, D. Mahalu, and H. Shtrikman, Science 290, 779 (2000); Y. Ji, M. Heiblum, and H. Shtrikman, Phys. Rev. Lett. 88, 076601 (2002).

${ }^{45}$ A. C. Hewson, The Kondo Problem to Heavy Fermions (Cambridge University Press, Cambridge, England, 1993), Chap. 7.

${ }^{46}$ L. I. Glazman and M. E. Raikh, Pis'ma Zh. Eksp. Teor. Fiz. 47, 378 (1988) [JETP Lett. 47, 452 (1988)]; T. K. Ng and P. A. Lee, Phys. Rev. Lett. 61, 1768 (1988).

${ }^{47}$ D. Goldhaber-Gordon, H. Shtrkman, D. Mahalu, D. AbuschMagder, U. Meirav, and M. A. Kastner, Nature 391, 156 (1998); S. M. Cronenwett, T. H. Oosterkamp, and L. P. Kouwenhoven, Science 281, 540 (1998); J. Schmid, J. Weis, K. Eberl, and K. von Klitzing, Physica B 256-258, 182 (1998).

${ }^{48}$ W. G. van der Wiel, S. De Franceschi, T. Fujisawa, J. M. Elzerman, S. Tarucha, and L. P. Kouwenhoven, Science 289, 2105 (2000).

${ }^{49}$ U. Gerland, J. von Delft, T. A. Costi, and Y. Oreg, Phys. Rev. Lett. 84, 3710 (2000). 
${ }^{50}$ B. R. Bulka and P. Stefanski, Phys. Rev. Lett. 86, 5128 (2001).

${ }^{51}$ W. Hofstetter, J. König, and H. Schoeller, Phys. Rev. Lett. 87, 156803 (2001).

${ }^{52}$ J. König and Y. Gefen, Phys. Rev. B 65, 045316 (2002).

${ }^{53}$ T.-S. Kim and S. Hershfield, Phys. Rev. Lett. 88, 136601 (2002).

${ }^{54}$ T. Rejec and A. Ramsak, Phys. Rev. B 68, 033306 (2003).

${ }^{55}$ P. G. Silvestrov and Y. Imry, Phys. Rev. Lett. 90, 106602 (2003).

${ }^{56}$ P. Simon, O. Entin-Wohlman, and A. Aharony, Phys. Rev. B 72, 245313 (2005).

${ }^{57}$ A. Aharony and O. Entin-Wohlman, Phys. Rev. B 72, 073311 (2005).

${ }^{58}$ G. D. Mahan, Many Particle Physics (Plenum, New York, 1990).
${ }^{59}$ I. G. Lang and Yu. A. Firsov, Zh. Eksp. Teor. Fiz. 43, 1843 (1962) [Sov. Phys. JETP 16, 1301 (1963)].

${ }^{60}$ G. Iche and A. Zawadowski, Solid State Commun. 10, 1001 (1972).

${ }^{61}$ A. Taraphder and P. Coleman, Phys. Rev. Lett. 66, 2814 (1991).

${ }^{62}$ A. M. Tsvelick and P. B. Wiegmann, Adv. Phys. 32, 453 (1983).

${ }^{63}$ K. G. Wilson, Rev. Mod. Phys. 47, 773 (1975).

${ }^{64}$ H. R. Krishna-murthy, J. W. Wilkins, and K. G. Wilson, Phys. Rev. B 21, 1003 (1980); 21, 1044 (1980).

${ }^{65}$ W. Hofstetter and G. Zarand, Phys. Rev. B 69, 235301 (2004).

${ }^{66}$ R. Žitko and T. Pruschke, Phys. Rev. B 79, 085106 (2009).

${ }^{67}$ D. C. Langreth, Phys. Rev. 150, 516 (1966). 